Title

\title{
Sigma Oscillations Protect or Reinstate Motor Memory Depending on their Temporal Coordination with Slow Waves
}

\section{Authors}

Judith Nicolas ${ }^{1,2,{ }^{*}}$, Bradley R. King ${ }^{3}$, David Levesque ${ }^{4}$, Latifa Lazzouni ${ }^{5}$, Emily Coffey ${ }^{6}$, Stephan Swinnen ${ }^{1,2}$, Julien Doyon ${ }^{5}$, Julie Carrier ${ }^{4,7}$, Geneviève Albouy ${ }^{1,2,3,{ }^{*}}$

\section{Affiliation}

${ }^{1}$ Department of Movement Sciences, Movement Control and Neuroplasticity Research Group, KU Leuven, 3001 Leuven, Belgium

${ }^{2}$ LBI - KU Leuven Brain Institute, KU Leuven, 3001 Leuven, Belgium

${ }^{3}$ Department of Health and Kinesiology, College of Health, University of Utah, Salt Lake City, UT 84112, USA

${ }^{4}$ Center for Advanced Research in Sleep Medicine, Centre Intégré Universitaire de Santé et de Services Sociaux du Nord-de-l'lle de Montréal, Montreal, QC, Canada

${ }^{5}$ McConnell Brain Imaging Centre, Department of Neurology and Neurosurgery, Montreal Neurological Institute, McGill University, Montreal, Quebec, Canada, H3A 2T5.

${ }^{6}$ Department of Psychology, Concordia University, Montreal, Quebec H4B 1R6, Canada

${ }^{7}$ Department of Psychology, Université de Montréal, Montreal, QC, Canada

\section{${ }^{*}$ Corresponding authors}

Judith Nicolas and Geneviève Albouy

Movement Control and Neuroplasticity Research Group

Department of Movement Sciences, KU Leuven

Tervuursevest 101 - Box 1501, 3001 Leuven, BELGIUM

Judith.nicolas@kuleuven.be; Genevieve.albouy@kuleuven.be 


\begin{abstract}
Targeted memory reactivation (TMR) during post-learning sleep is known to enhance motor memory consolidation but the underlying neurophysiological processes remain unclear. Here, we confirm the beneficial effect of auditory TMR on motor performance. At the neural level, TMR enhanced slow wave (SW) characteristics. Additionally, greater TMR-related phase-amplitude coupling between slow (0.5-2 $\mathrm{Hz})$ and sigma $(12-16 \mathrm{~Hz})$ oscillations after the SW peak was related to higher TMR effect on performance. Importantly, sounds that were not associated to learning strengthened SW-sigma coupling at the SW trough and the increase in sigma power nested in the trough of the potential evoked by these unassociated sounds was related to the TMR benefit. Altogether, our data suggest that, depending on their precise temporal coordination during post learning sleep, slow and sigma oscillations play a crucial role in either memory reinstatement or protection against irrelevant information; two processes that critically contribute to motor memory consolidation.
\end{abstract}

\title{
Key words
}

Motor learning, Targeted Memory Reactivation, Memory Consolidation, Sleep, Slow waves, Spindles, Sigma oscillations, Slow oscillations 


\section{Introduction}

Motor memory is the capacity that affords the development of a repertoire of motor skills essential for daily life activities such as typing on a keyboard or buttoning a shirt. After initial acquisition, a motor memory undergoes consolidation, which is the offline process (i.e., without further practice) by which the acquired memory trace becomes stable and long-lasting $(1,2)$. Sleep, and non-rapid eye movement sleep (NREM) in particular $(3,4)$, is thought to offer a privileged window for the consolidation process to occur (5). The specific electrophysiological events characterizing NREM sleep, such as slow waves (SW - high amplitude waves in the $0.5-2 \mathrm{~Hz}$ frequency band) (6), thalamo-cortical spindles (short burst of oscillatory activity in the $12-16 \mathrm{~Hz}$ sigma band) $(7,8)$ and hippocampal ripples (80-100 Hz oscillations in humans) (9), as well as their precise synchrony, have been described to support neuroplasticity processes underlying consolidation (10).

In recent years, there has been increasing evidence in both the declarative and motor memory domains that memory consolidation processes can be augmented by experimental interventions such as targeted memory reactivation (TMR) applied during post-learning sleep $(11,12,13,14)$. In TMR protocols, sensory stimuli (e.g., sounds) that are associated to the learned material during the learning episode are presented offline, during the consolidation interval, in order to reactivate the encoded memory trace (6). This memory reinstatement is thought to be supported by a TMR-mediated reinforcement of the endogenous brain reactivation patterns that occur spontaneously during the consolidation process. Such reactivations are thought to support the transfer of memory traces to the neocortex (15). While the beneficial effect of TMR on motor performance has been highlighted in previous research (e.g. 16) $(17,13,12)$, the neurophysiological processes supporting these effects have been scarcely studied. Therefore, the goal of the present study was to elucidate the neurophysiological processes supporting memory reactivation during sleep which underlie TMR-induced enhancement in motor memory consolidation.

We designed a within-participant experiment (Figure 1) pre-registered in the Open Science Framework (available at osf.io/n3me8). Young healthy participants were trained on a Serial Reaction Time task (22, 23) during which they learned two different motor sequences, each associated to a particular sound. Participants were then offered a 90-minute nap that was monitored with polysomnography. During NREM 2-3 sleep stages, the sound associated to one of the two trained sequences ('Associated' sound to the 'Reactivated' sequence) as well as a control sound ('Unassociated') that was not associated to the learned material were played. The sound associated to the other learned sequence was not presented during the nap, thus serving as a no-reactivation control condition ('Non-reactivated'). The time course of the TMRinduced consolidation process was assessed with retests after the nap episode as well as after a night of sleep spent at home. At the behavioral level, results demonstrated the expected TMR benefit. At the brain level, they indicate a TMR-mediated enhancement of SWs and SW-sigma coupling after the peak of the SW such that the higher the coupling, the greater the effect of TMR on motor performance. Intriguingly, unassociated sounds also strengthened SW-sigma coupling but at a different phase of the SW (trough) and the increase in sigma power nested in the trough of the potential evoked by the unassociated sounds was related to the TMR benefit. Altogether, our findings suggest that sigma oscillations may play a dual role in the consolidation process depending on both the nature of the information to be processed and the phase of the slow oscillation in which they occur. We propose that sigma oscillations protect or reinstate motor memory depending on their temporal coordination with slow oscillations during post-learning sleep. 


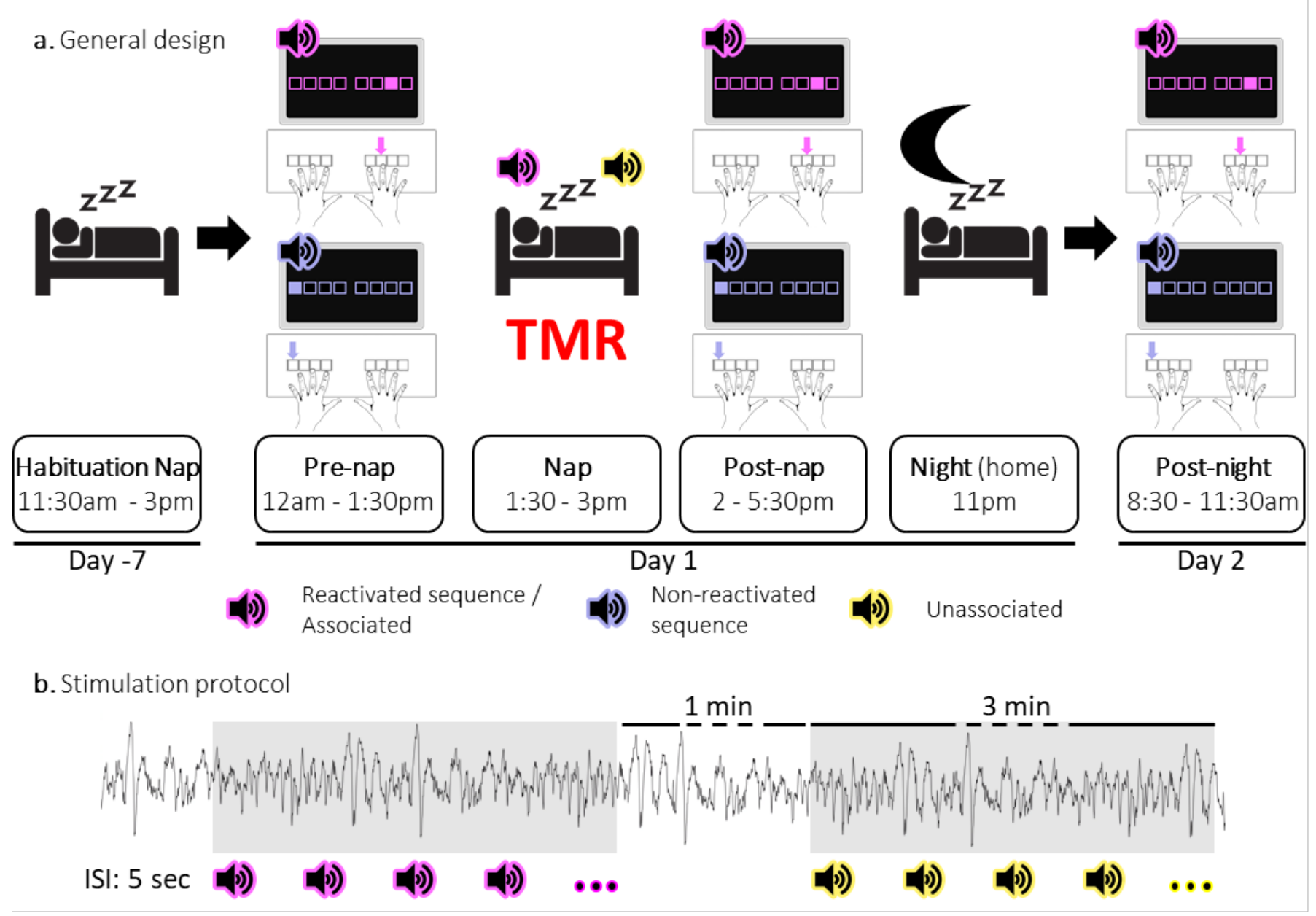

Figure 1: Experimental protocol. a. General design. Following a habituation nap that was completed approximately one week prior to the experiment, participants underwent a pre-nap motor task session, a 90-minute nap episode monitored with polysomnography during which targeted memory reactivation (TMR) was applied and a post-nap retest session. Participants returned to the lab the following morning to complete an overnight retest (post-night). During the motor task, two movement sequences were learned simultaneously and were cued by two different auditory tones. For each movement sequence, the respective auditory tone was presented prior to each sequence execution (i.e., one tone per sequence). One of these specific sounds was replayed during the subsequent sleep episode (Reactivated) and the other one was not (Non-reactivated). During the NREM 2-3 stages of the post-learning nap, two different sounds were presented. One was the sound associated (Associated) to one of the previously learned sequences, i.e. to the reactivated sequence, and one was novel, i.e., not associated to any learned material (Unassociated). b. Stimulation protocol. Stimuli were presented during three-minute stimulation intervals of each cue type alternating with a silent 1-minute period (rest intervals). The inter-stimulus interval (ISI) was of $5 \mathrm{sec}$. The stimulation was manually stopped when the experimenter detected REM sleep, NREM1 or wakefulness. 
bioRxiv preprint doi: https://doi org/10.1101/2021.09.02 458683; this version posted November $12,2021$. The copyright holder for this preprint (which was not certified by peer review) is the author/funder, who has granted bioRxiv a license to display the preprint in perpetuity. It is made available under aCC-BY 4.0 International license.

\section{Results}

The analyses presented in the current paper that were not pre-registered are referred to as exploratory.

\subsection{Behavioral data}

As per our pre-registration, behavioral analyses focused on performance speed (i.e., response time (RT) on correct key presses) on the motor sequence learning task measured at three time points: pre-nap, postnap and post-night (Figure 1a).

Analyses of the pre-nap training data indicated that participants learned the two sequence conditions (reactivated and non-reactivated sequences) to a similar extent during initial learning (16 blocks of training; main effect of Block: $F(15,345)=34.82 ; p$-value $=2.04 \mathrm{e}-26 ; \eta^{2}=0.6$; main effect of Condition: $F(1,23)=0.16 ; p$-value $=0.69$; Block by Condition interaction: $F(15,345)=1.09 ; p$-value $=0.37$; Figure 2a). After initial training, participants were offered a short break ( $\sim$ minute) and were then tested again on the learned motor sequences. This short pre-nap test session was designed to offer a fatigue-free measure of end-of-training, asymptotic performance to be used as baseline for the computation of subsequent offline changes in performance (see description below) (24). Before computing offline performance gains, we first assessed whether participants reached stable and similar performance levels between conditions during the pre-nap test session. Results showed that while performance reached similar levels between conditions (4 blocks; main effect of Condition: $F(1,23)=3.39 e-5$; $p$-value $=0.99$; Block by Condition interaction: $F(3,69)=1.21 ; p$-value $=0.31)$, asymptotic performance levels were not reached as shown by a significant Block effect $\left(F(3,69)=6.67 ; p\right.$-value $\left.=0.001 ; \eta^{2}=0.22\right)$. To meet the performance plateau prerequisite to compute offline gains in performance, the first block of the pre-nap test session driving this effect was removed from further analyses. Performance on remaining blocks was stable as indicated by a non-significant Block effect $(F(2,46)=1.56 ; p$-value $=0.22)$. Similar to above, the main effect of Condition $(F(1,23)=0.04 ; p$-value $=0.85)$ and the Block by Condition interaction $(F(2,46)=1.81 ; p$-value $=0.18)$ were not significant. Altogether, these results indicate that a performance plateau was ultimately reached and both sequence conditions were learned similarly (Figure 2a).

Post-nap and post-night offline gains in performance were then computed for both conditions as the relative change in speed between the three plateau blocks of the pre-nap test and the first four blocks of the post-nap and post-night sessions, respectively. A repeated measures analysis of variance (rmANOVA) performed on offline gains in performance with Time-point (post-nap vs. post-night) and Condition (reactivated vs. non-reactivated) as within-subject factors showed a significant Time-point effect, whereby gains were significantly higher at the post-night as compared to the post-nap retest $(F(1,23)=46.53$; $p$ value $=5.89 \mathrm{e}-7 ; \eta^{2}=0.67$; Figure $2 \mathrm{~b}$ ). Critically, offline gains for the reactivated sequence were significantly higher than for the non-reactivated sequence (Condition effect: $F(1,23)=4.75 ; p$-value $=0.0397 ; \eta^{2}=0.17$ ). The Condition by Time-point interaction was not significant $(F(1,23)=7.42 \mathrm{e}-4 ; p$-value $=0.98)$. In conclusion, our behavioral results indicate a TMR-induced enhancement in performance that did not differ across nap and night intervals. 


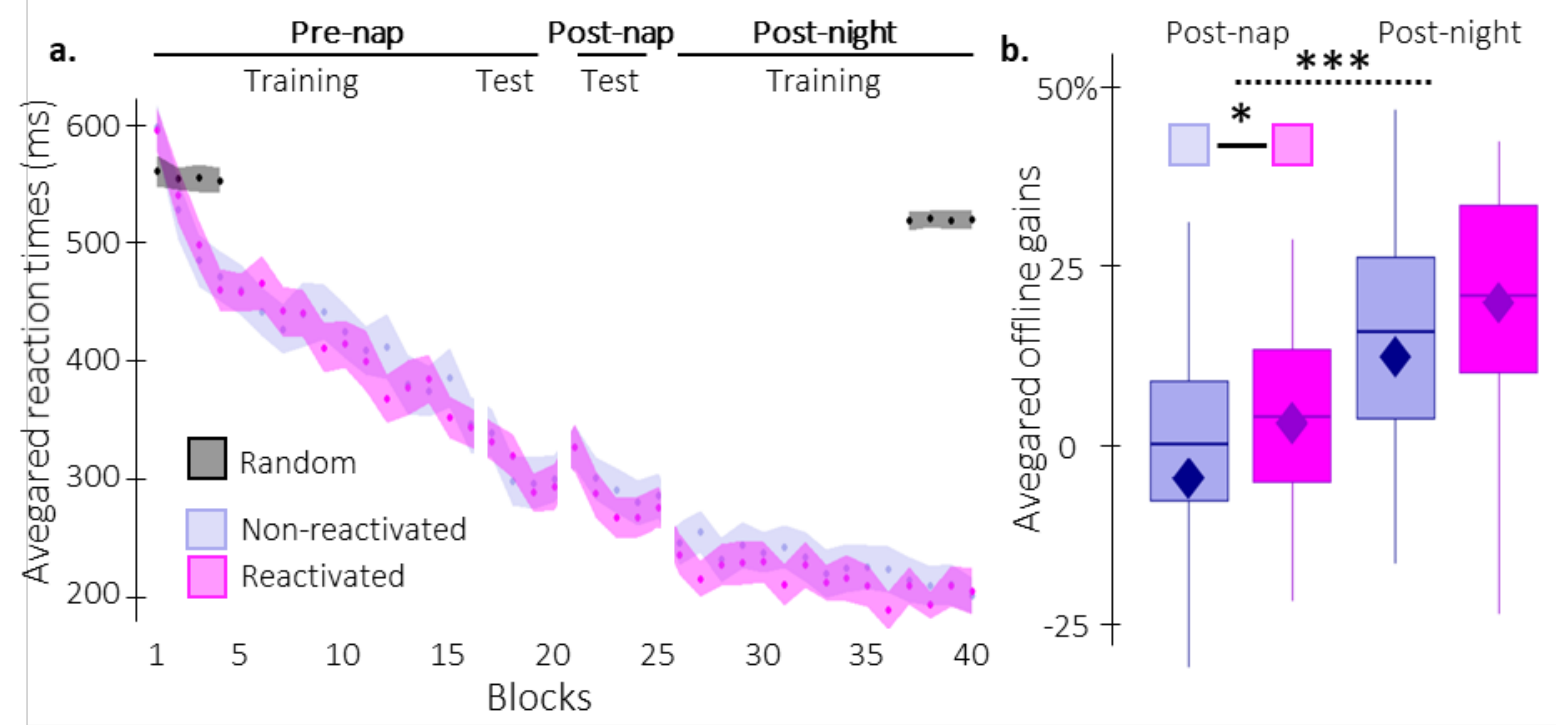

Figure 2: Behavioral results. a. Performance speed (mean reaction time in ms) across participants plotted as a function of blocks of practice during the pre-and post-nap sessions (+/-standard error in shaded regions) for the reactivated (magenta) and the non-reactivated (blue) sequences and for the random SRTT (Black overlay). $b$. Offline gains in performance speed (\% change) averaged across participants (box: median (horizontal bar), mean (diamond) and first(third) as lower(upper) limits; whiskers: 1.5 x InterQuartile Range (IQR)) for post-nap and post-night time-points and for reactivated (magenta) and non-reactivated (blue) sequences. The results highlighted a main effect of Timepoint (***: p-value <0.001) and a main effect of Condition (*: p-value <0.05).

\subsection{Electrophysiological data}

Participants' sleep was recorded using a 6-channel EEG montage during a 90min episode following learning. Sleep was monitored online and sounds were presented during NREM sleep stages. Sleep characteristics resulting from the offline sleep scoring as well as the distribution of auditory cues across sleep stages are shown in Table 1. Results indicate that all the participants slept well and that cues were accurately presented in NREM sleep.

Table 1. Sleep and stimulation characteristics $(N=24)$

\section{Daytime sleep characteristics}

Time allowed to sleep

Total Sleep Time ${ }^{a}$

Sleep Efficiency ${ }^{b}$

NREM1 Latency

Time awake

Time in NREM1 Sleep

Time in NREM2 Sleep

Time in NREM3 Sleep

Time in REM Sleep

\section{Participants reaching}

NREM3 sleep

REM sleep

Number of Auditory cues

During all stages

During wake

During NREM1 Sleep

During NREM2-3 Sleep

During REM Sleep

Accuracyc
$89.7 \min [88.5$ - 90.9]

$67 \min [61.1-73]$

$74.9 \%$ [68.1-81.8]

$4.5 \min [3.4-5.6]$

$10.6 \min [6.9-14.3]$

$12.1 \mathrm{~min}[8.6-15.5]$

$39.9 \min [33.8-46.1]$

$17.5 \min [8.7-26.2]$

$9.6 \min [5.8-13.4]$

$\mathrm{N}=20$

$N=18$

All cues

389 [339-439]

8.8 [4.1-13.5]

$22.1[9.1-35.1]$

349.5 [293.8 - 405.2]

8.5 [3.6- 13.5]

$88.4 \%$ [82.9-93.9]
Associated cues

192.5 [167.1 - 218.1]

$3.8[1.2-6.4]$

$11.6[4.7-18.5]$

173.4 [145.3 - 201.5]

$3.8[1-6.5]$

$88.7 \%$ [82.8-94.6]
Unassociated cues

196.4 [171.5 - 221.3]

$5[1.8-8.2]$

$10.5[3.9-17.1]$

176.1 [147.9 - 204.3]

$4.8[1.7-7.9]$

$88.2 \%$ [82.3 - 94] 
bioRxiv preprint doi: https://doi org/10.1101/2021.09.02.458683; this version posted November $12,2021$. The copyright holder for this preprint (which was not certified by peer review) is the author/funder, who has granted bioRxiv a license to display the preprint in perpetuity. It is made available under aCC-BY 4.0 International license.

Notes. Values are means [lower and upper limit of the 95\% Confidence Interval - Cl]. REM: Rapid Eye Movement. ${ }^{a}$

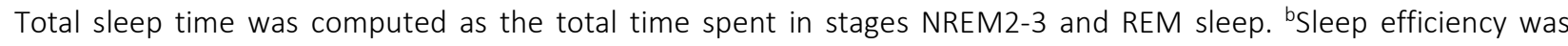
computed as the percent of time asleep (namely in NREM2-3 and in REM sleep) relative to the total time in bed (specifically, from lights off to lights on). ' Percentage of auditory cues correctly sent during NREM2 and NREM3 sleep.

\subsubsection{Event-related analyses}

First, auditory evoked potentials were computed across all EEG channels (but see supplemental Figure S3 for channel level data) separately for associated and unassociated auditory cues presented during NREM23 stages (18) (Figure 3a). ERP amplitude was extracted for the 2 conditions from the temporal window highlighted in Figure $3 a$ in which the amplitude of the auditory responses across conditions was significantly lower (trough) than zero (from 0.44 to $0.63 \mathrm{sec}$ relative to cue onset, see methods, supplemental results and supplemental Figure S2). Between-condition comparisons using Wilcoxon signed-rank test showed that the amplitude of the ERP trough was significantly deeper $(V=75, p$-value $=$ 0.016) following associated as compared to unassociated cues (Figure 3b).

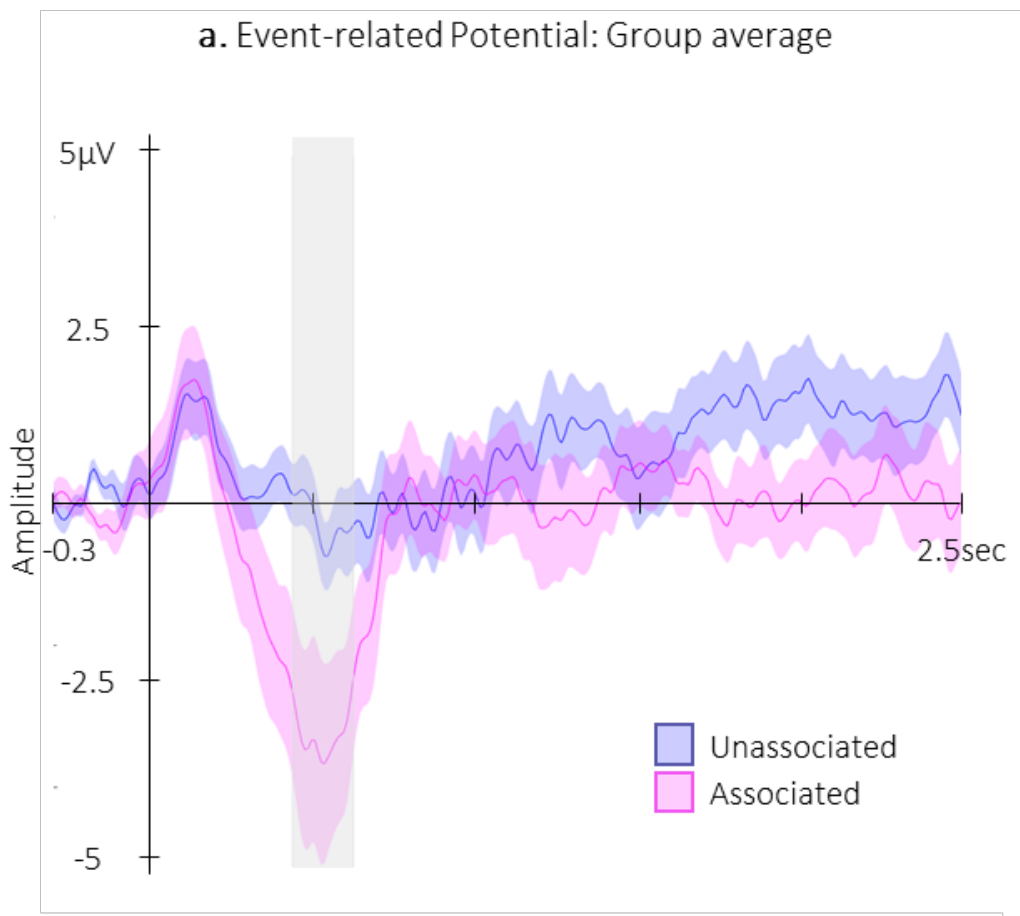

\section{b. Trough amplitude $(0.44-0.63 \mathrm{~s})$}

Figure 3: Event-related Potentials (ERP). a. Potentials averaged across all participants and all EEG channels (+/standard error in shaded regions) evoked by the associated (magenta) and the unassociated (blue) auditory cues from -0.3 to $2.5 \mathrm{sec}$ relative cue onset. The grey region represents the temporal window (trough) in which ERPs across conditions were significantly different from zero. b. ERP amplitude (box: median (horizontal bar), mean (diamond) and first(third) as lower(upper) limits; whiskers: $1.5 \times 1 Q R$ ) extracted from the temporal window highlighted in panel a, i.e., at $0.44-0.63$ sec post-cue onset (trough) in each condition. *: p-value $<0.05$

Next, we investigated whether EEG sigma oscillation power $(12-16 \mathrm{~Hz})$ evoked by the auditory cues across all EEG channels was modulated by the different stimulation conditions in the $2.5 \mathrm{sec}$ following the cue onset. Note that, for completeness, time-frequency analyses were performed on a wider frequency range $(5-30 \mathrm{~Hz})$ and that analyses outside the sigma band were considered as exploratory. Cluster Based Permutations (25) (CBP) tests did not highlight any clusters between the two auditory cues. 


\subsubsection{Sleep event detection}

Slow waves (SWs) and spindles were detected automatically (26) on all EEG channels in all NREM2-3 sleep epochs (thus including associated and unassociated sound stimulation intervals as well as non-stimulation intervals, see Figure 1b). The detection tool identified on average 424.8 [ $95 \% \mathrm{Cl} 328-521.6$ ] slow waves and 98 [95\% Cl: $82.8-113.2$ ] spindles averaged across channels during the nap episode (see methods for details on the detection algorithms and supplemental Table S3 for the number of events detected on each channel and each condition).

Concerning the detected SWs (Figure 4a), both peak-to-peak (PTP) amplitude and density were greater for the associated as compared to the unassociated stimulation intervals (amplitude: $t=2.7 ; \mathrm{df}=21 ; \mathrm{p}$-value $=0.009$; Cohen's $d=0.55$; and density $V=197 ; p$-value $=0.01 ; r=0.58)$. Exploratory analyses including the detected SWs in the non-stimulation (rest) intervals did not highlight PTP amplitude differences between the rest intervals and the two types of stimulation intervals (rest vs. associated: $t=0.82 ; \mathrm{df}=21 ; \mathrm{p}$-value $=0.42$; rest vs. unassociated: $t=-0.92 ; d f=21 ; p$-value $=0.42 ;$ Figure $4 b-c)$. However, $S W$ density was significantly lower during the rest as compared to the stimulation intervals, regardless of the cue type (rest vs. associated: $V=232 ; p$-value $=0.0002 ; r=0.66$; rest vs. unassociated: $V=224 ; p$-value $=0.0008 ; r=0.6$; Figure $4 d$ ). Altogether, these results indicate that auditory stimulation induced an overall increase in SW density, and, more importantly, that the associated sounds resulted in an increase in SW amplitude, density and slope as compared to the unassociated sounds.

Sleep spindle density did not differ between associated and unassociated stimulation intervals $(V=98, p-$ value $=0.89$ ). Similarly, exploratory analyses on additional spindle features including amplitude and frequency did not yield any significant differences between stimulation conditions (all p-values $>0.2$ ). As no effect of stimulation condition was observed on spindle characteristics, conditions were pooled together in exploratory analyses including spindles detected during rest intervals (Figure 5). Results show that spindle density did not differ between stimulation and rest intervals ( $V=97, p$-value $=0.22$ ). Interestingly, spindle amplitude was significantly higher during the auditory stimulation intervals as compared to the rest intervals $(V=232 ; p$-value $=0.003 ; r=1.04)$, whereas spindle frequency showed the opposite pattern ( $t=-3.42 ; d f=22 ; p$-value $=0.005$; Cohen's $d=0.71)$. In summary, these results indicate that while auditory stimulation altered spindle features as compared to rest, the two sound conditions did not differently influence spindle characteristics. 


\section{a. Detected slow waves: Group average}

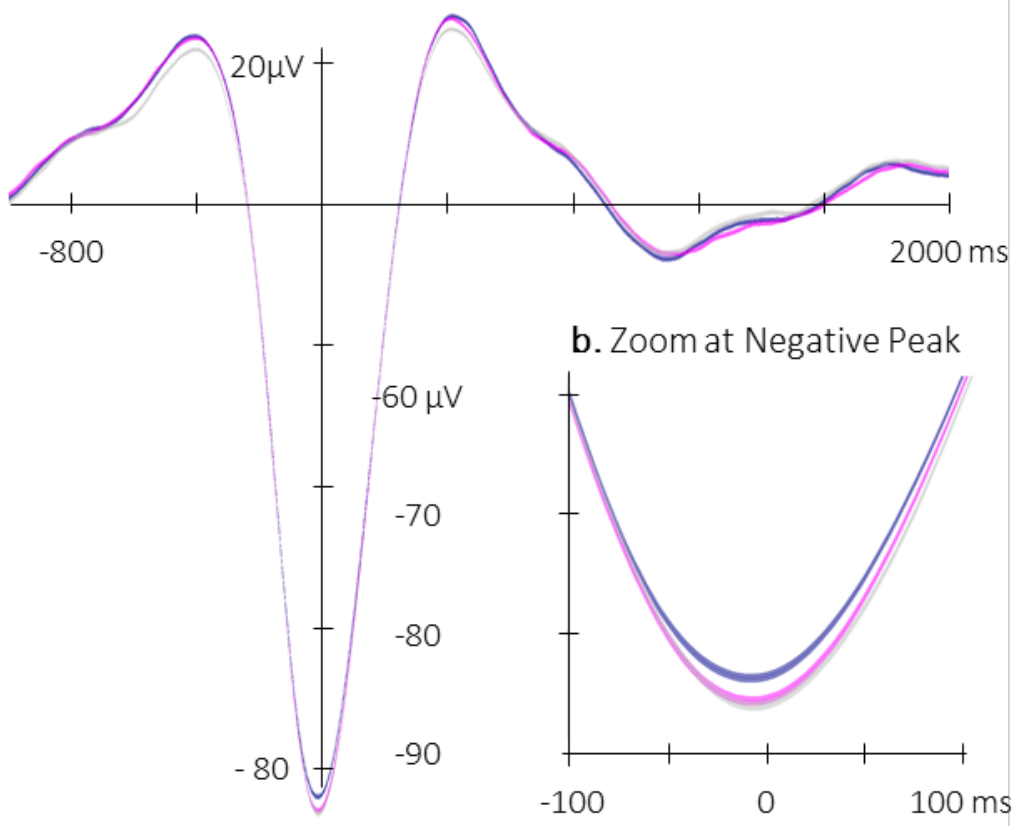

c. Peak-to-Peak

d. Density
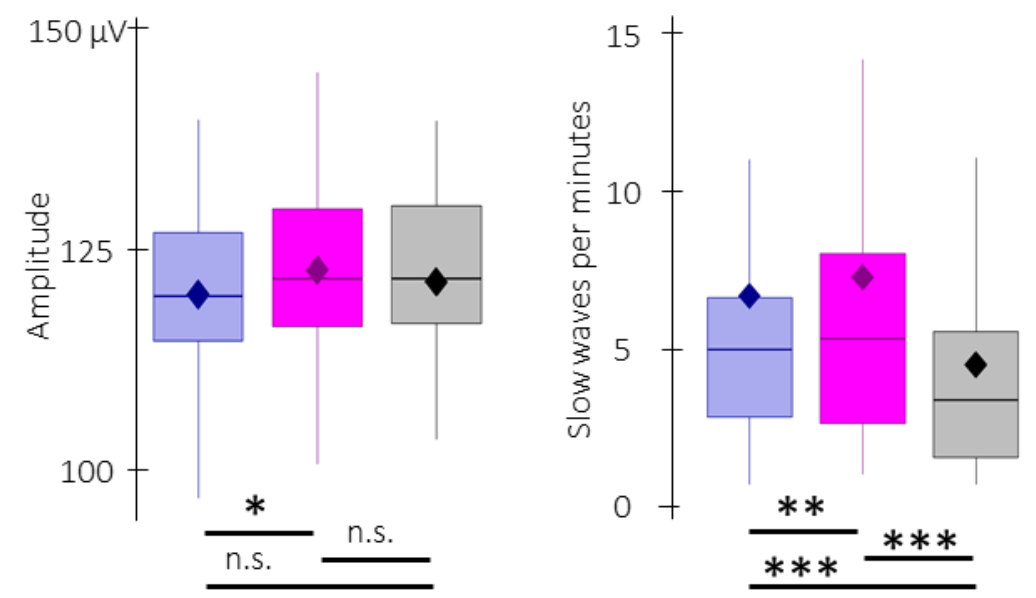

Conditions

Rest

Associated

Unassociated

Figure 4: Detected Slow Waves (SWS). a. Average at the negative peak (+/-standard error) across all detected slow waves during the associated (magenta) and unassociated (blue) stimulation intervals as well as in the rest (i.e. unstimulated) intervals (grey). b. Zoom on the negative peak of the detected SWs. Shaded regions represent SEM. $c$. Peak-to-peak SW amplitude ( $\mu \mathrm{V})$ was higher for associated as compared to unassociated sounds. $d$. SW density (number of SWs per total time in minutes spent in stimulation or rest intervals) was higher during stimulation as compared to rest intervals and for associated as compared to unassociated sounds. Box: median (horizontal bar), mean (diamond) and first(third) as lower(upper) limits; whiskers: $1.5 \times 1 Q R$; *: p-value <0.05; **: p-value <0.01; ***: p-value $<0.001$; n.s.: not significant. 


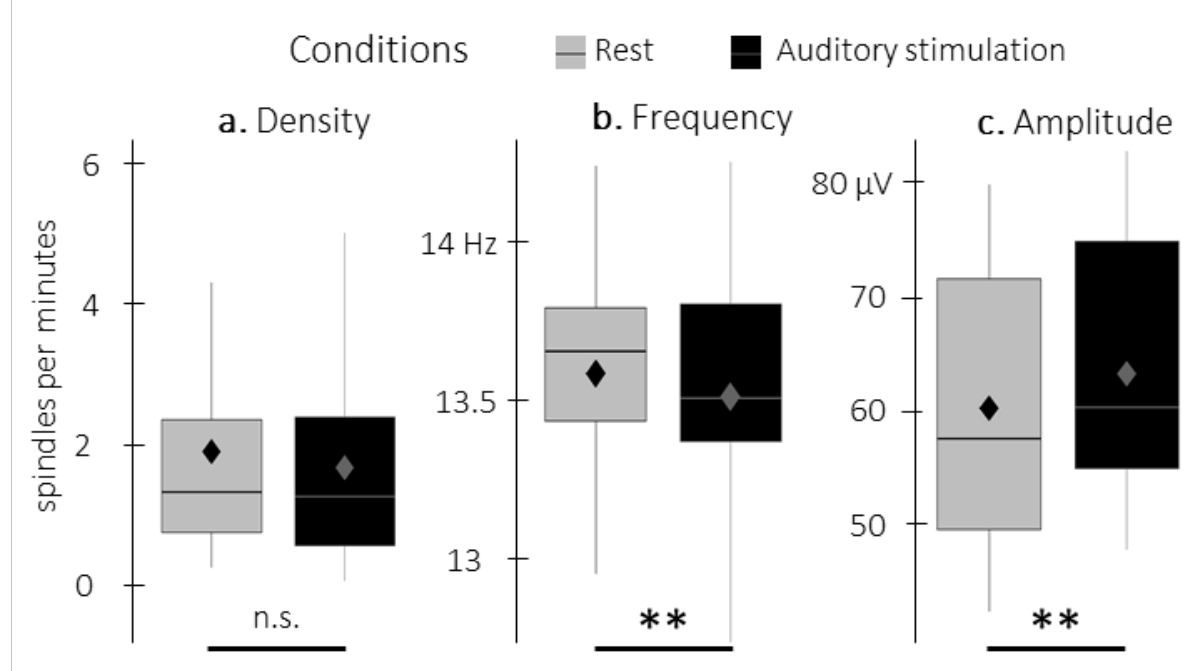

Figure 5: Detected spindles. a. Spindle density (number of spindles per total time in minutes spent in stimulation or rest intervals) did not differ between stimulation intervals (irrespective of sound type; black) and rest (grey) intervals. b. Spindle frequency $(\mathrm{Hz})$ was lower during stimulation as compared to rest intervals. c. Spindle amplitude $(\mu \mathrm{V})$ was higher during stimulation as compared to rest intervals. Box: median (horizontal bar), mean (diamond) and first(third) as lower(upper) limits; whiskers: $1.5 \times$ IQR; ${ }^{* *}$ : $p$-value <0.01; n.s.: not significant.

\subsubsection{Phase-amplitude coupling}

We investigated whether the phase of the slow oscillations in the $0.5-2 \mathrm{~Hz}$ frequency band was coupled to the amplitude of sigma $(12-16 \mathrm{~Hz})$ oscillations following either the auditory cue or the negative peak of the detected (i.e., spontaneous) SWs. Similar to above, phase-amplitude coupling (PAC) analyses were performed on a wider frequency range $(7-30 \mathrm{~Hz})$ for completeness; thus, analyses outside the preregistered sigma band (see red frame in Figure 6) are considered exploratory.

The PAC values locked to the auditory cues were compared between the two stimulation conditions. The CBP test did not highlight any significant clusters (alpha threshold $=0.025$, cluster p-value $=0.44$ ). The preferred coupling phase (Table 2), which represents the phase at which the maximum amplitude is observed, did not significantly differ between conditions (Watson's $U^{2}=0.06$ ). These results suggest that the stimulation conditions did not influence the coupling between the phase of the slow oscillations and the amplitude of sigma oscillations at the auditory cue.

Table 2. Group average (+/- $95 \%$ confidence interval) of the preferred phase

\begin{tabular}{c|c|c|c|c}
\multicolumn{2}{c|}{ Cue-locked Preferred Phase } & \multicolumn{3}{|c}{ sW-locked Preferred Phase } \\
\hline Associated & Unassociated & Associated & Unassociated & Rest \\
$-0.34[-0.36-0.32]$ & $-0.11[-0.13-0.09]$ & $3.16[3.15-3.17]$ & $2.74[2.73-2.75]$ & $3.02[3.02-3.03]$
\end{tabular}


bioRxiv preprint doi: https://doi.org/10.1101/2021.09.02.458683; this version posted November $12,2021$. The copyright holder for this preprint (which was not certified by peer review) is the author/funder, who has granted bioRxiv a license to display the preprint in perpetuity. It is made available under aCC-BY 4.0 International license.

Comparison of the PAC locked to the negative peak of the SWs (Figure 6) between stimulation conditions revealed a significant cluster (alpha threshold $=0.025$, cluster $p$-value $=0.024$; Cohen's $d=-0.56$ ). Specifically, the coupling between the phase of the signal in the $0.5-2 \mathrm{~Hz}$ frequency band and the amplitude of the signal in the 14-18 Hz frequency band was significantly stronger around the negative SW peak (from -0.8 to $0.2 \mathrm{sec}$ relative to negative peak) during unassociated as compared to associated stimulation intervals (Figure 6b). The exploratory comparison between rest and associated stimulation intervals did not reveal any significant clusters (alpha threshold $=0.025$, all cluster $p$-values $>0.6$ ) but a significant cluster was observed between unassociated stimulation and rest (alpha threshold $=0.025$, cluster $p$-value $=0.001$; Cohen's $d=0.53$; Figure $6 c$ ). This cluster was observed between 13.5 and $20 \mathrm{~Hz}$ and -1 to $0.5 \mathrm{sec}$ around the negative peak of the SW. The preferred phases in each of the conditions were not significantly different (associated vs. unassociated: Watsons $U^{2}=0.15$; associated vs. rest: Watsons $U^{2}=0.05$; unassociated vs. rest: Watsons $U^{2}=0.1$ ). Altogether, these results suggest that slow-sigma oscillation coupling observed just before the onset of the SW was stronger during unassociated as compared to associated and rest intervals but that the preferred coupling phase was not modulated by the experimental conditions.

\section{a. Slow Wave locked phase amplitude coupling}

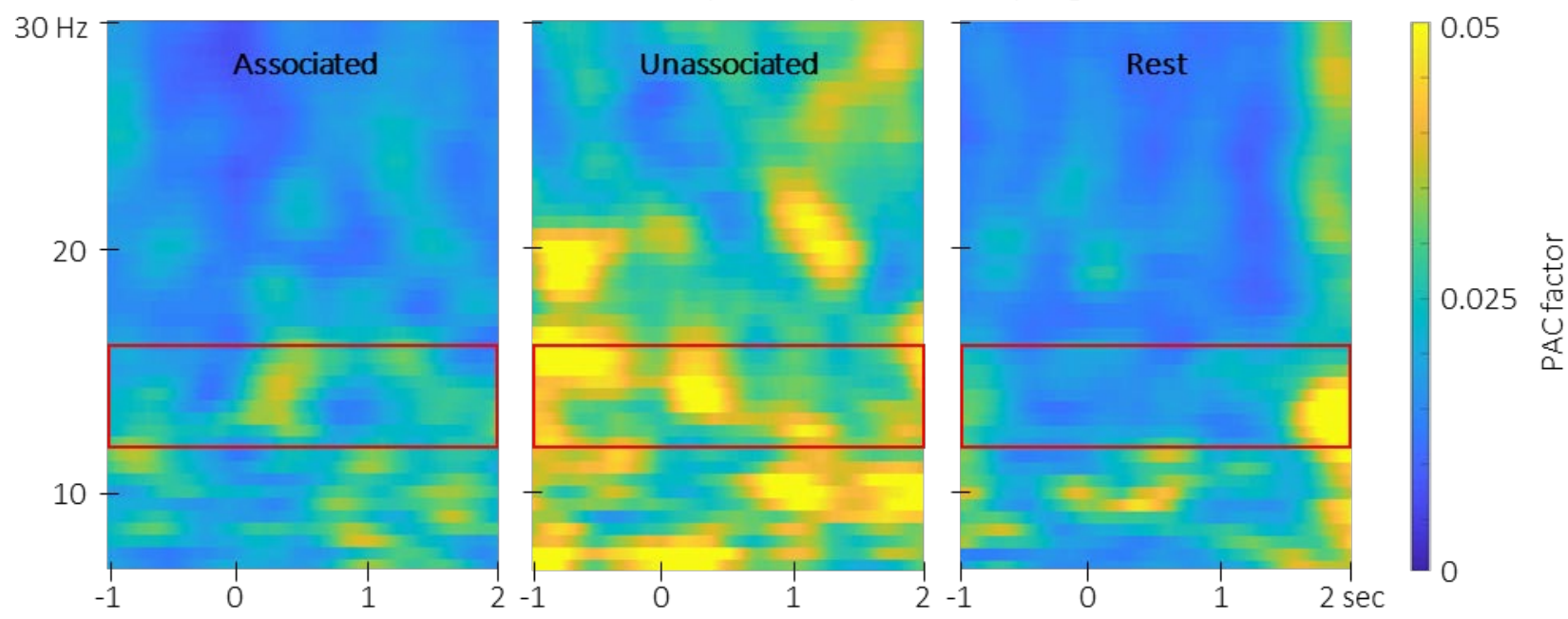

b. Unassociated - Associated

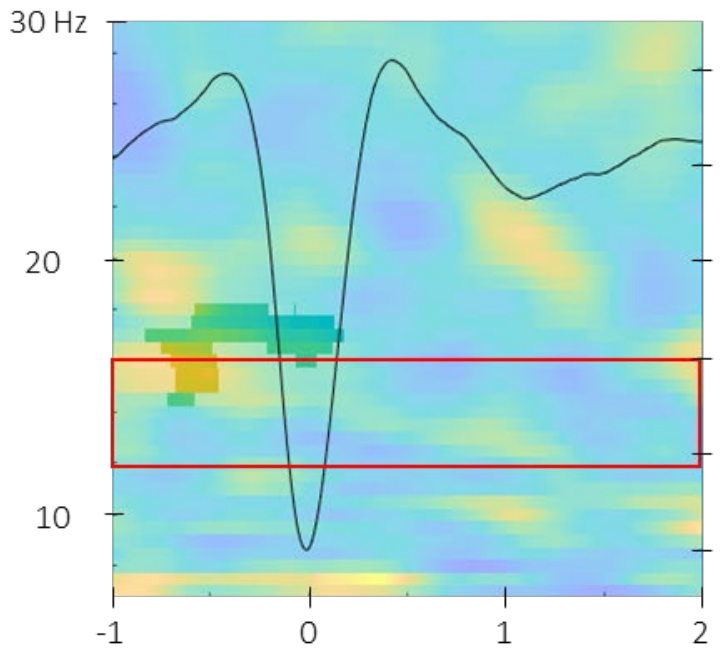

c. Unassociated - Rest

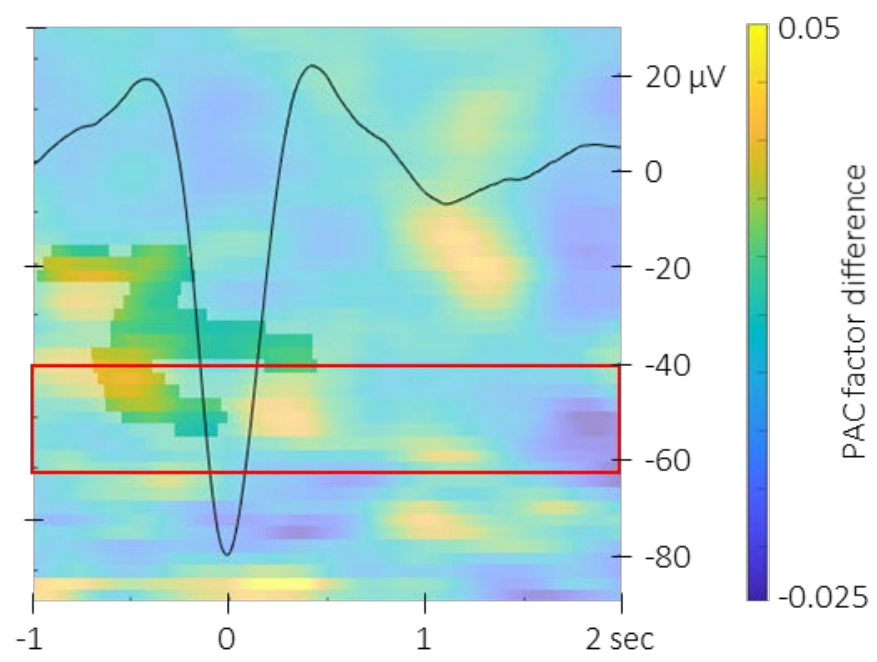

Figure 6: Phase-amplitude coupling locked to the detected slow wave negative peaks. a. Time-Frequency Representation (TFR) of group average coupling strength between the phase of the $0.5-2 \mathrm{~Hz}$ frequency band and the amplitude from 7 to $30 \mathrm{~Hz}$ (y-axis) from -1 to $2 \mathrm{sec}$ (x-axis) relative to SW negative peak for the three interval types. b. PAC was significantly higher during the unassociated as compared to the associated sound intervals in the highlighted cluster. c. PAC was significantly higher during the unassociated sound as compared to the rest intervals in the highlighted cluster. Red frames indicate the pre-registered sigma frequency band of interest. Superimposed on the TFR in panels b and c (black line): SW grand average across individuals and conditions (y-axis on right). 
bioRxiv preprint doi: https://doi.org/10.1101/2021.09.02.458683; this version posted November 12, 2021. The copyright holder for this preprint (which was not certified by peer review) is the author/funder, who has granted bioRxiv a license to display the preprint in perpetuity. It is made available under aCC-BY 4.0 International license.

\subsection{Correlational analyses}

Correlation analyses between the TMR index (i.e., the difference in offline gains in performance between the reactivated and the non-reactivated sequence) and the density of SW and spindles as well as with the amplitude of the ERP did not yield any significant results (see supplemental results). However, the correlational CBP analysis between the TMR index and the difference in TF power elicited by the different auditory cues highlighted one significant cluster (alpha threshold $=0.025$, cluster centered on 0.5 sec. postcue $p$-value $=0.022$, rho $=-0.46$; Figure $7 a$ ). For illustration purposes, we extracted the difference in TF power within the pre-registered sigma band and in the 0.44 and $0.66 \mathrm{sec}$ window in which the amplitude of the ERP trough differed between conditions (see Figure 3 ). The resulting scatter plot presented in Figure $7 \mathrm{~b}$ indicates that higher TMR index (i.e., greater behavioral benefit of TMR) was related to higher sigma oscillation power for the unassociated compared to the associated sound condition (exploratory two-sided Spearman correlations: $S=3374, p$-value $=0.023 ; r=-0.47$ ).

\section{a. Associated-Unassociated}

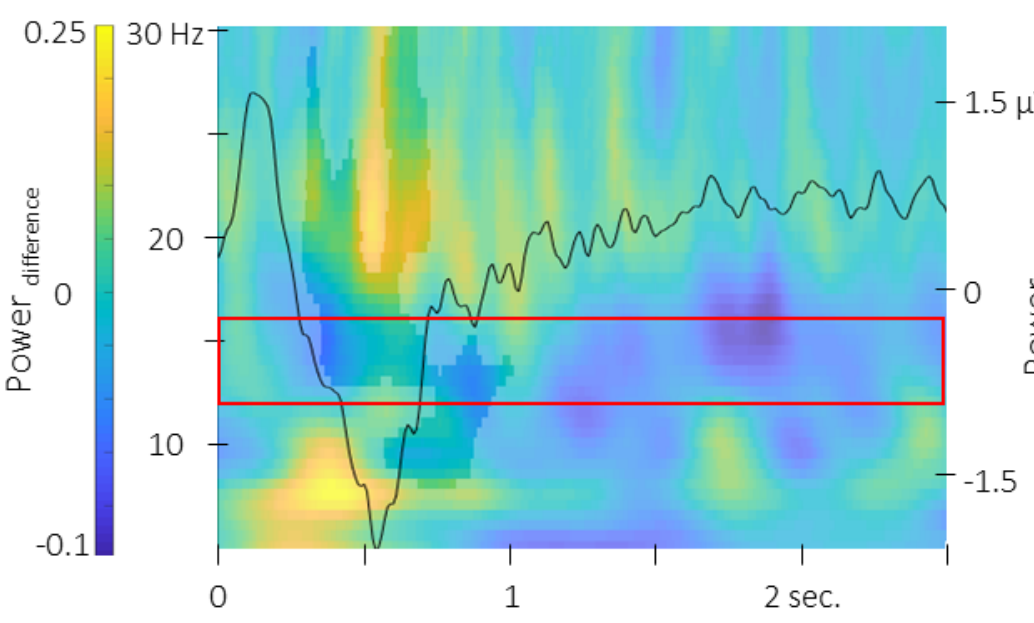

\section{b. Correlation between} sigma power difference and TMR index

Figure 7: Correlation between power difference and TMR Index. a. Time-Frequency Representation (TFR) of the difference between the power elicited by the associated auditory cues and the unassociated ones correlated with the $T M R$ index. Highlighted, the negative clusters in which the TMR index is significantly correlated with the difference in power. Red frame indicates the pre-registered sigma frequency band of interest. Superimposed on the TFR (black line): Grand average across individuals and conditions of event related potentials elicited by the auditory cues ( $y$-axis on right). $b$. Negative correlation between the power difference (0.86-1.06 sec post-cue, 12-16 Hz) and the TMR index (dots represent individual datapoints).

Finally, with respect to PAC-TMR index correlation analyses, no significant correlation was observed between the auditory-locked PAC metrics and the TMR index (alpha threshold $=0.025$, cluster $p$-values $>$ 0.09). In contrast, cluster-based permutation correlational tests performed between the $12-16 \mathrm{~Hz}$ TFR SWlocked PAC difference between the two conditions and the TMR index revealed a significant cluster. Results show that the associated vs. unassociated difference in coupling strength between the phase of the signal in the $0.5-2 \mathrm{~Hz}$ frequency band and the amplitude of the signal in the 14.5-17 Hz frequency band, just after the SW peak $(0.5$ and $1 \mathrm{sec}$ ), was positively correlated with the TMR index (alpha threshold $=0.025$, cluster $\mathrm{p}$-value $=0.0499$, rho $=0.55$; Figure 8a). For illustration purposes, we extracted the difference in PAC at the center of the significant cluster (from 0.68 to $0.82 \mathrm{sec}$ and between 14.5 and $17 \mathrm{~Hz}$ ). The resulting scatter plot (Figure $8 \mathrm{~b}$ ) indicates that the stronger the phase-amplitude coupling during associated as compared to the unassociated stimulation intervals, the higher the TMR index (exploratory two-sided 
bioRxiv preprint doi: https://doi.org/10.1101/2021.09.02.458683; this version posted November 12, 2021. The copyright holder for this preprint (which was not certified by peer review) is the author/funder, who has granted bioRxiv a license to display the preprint in perpetuity. It is made available under aCC-BY 4.0 International license.

Spearman correlations: $S=826, p$-value $=0.012 ; r=0.5$; without the extreme - albeit non-outlier participant: $S=826, p$-value $=0.036$ ).

a. Associated- Unassociated

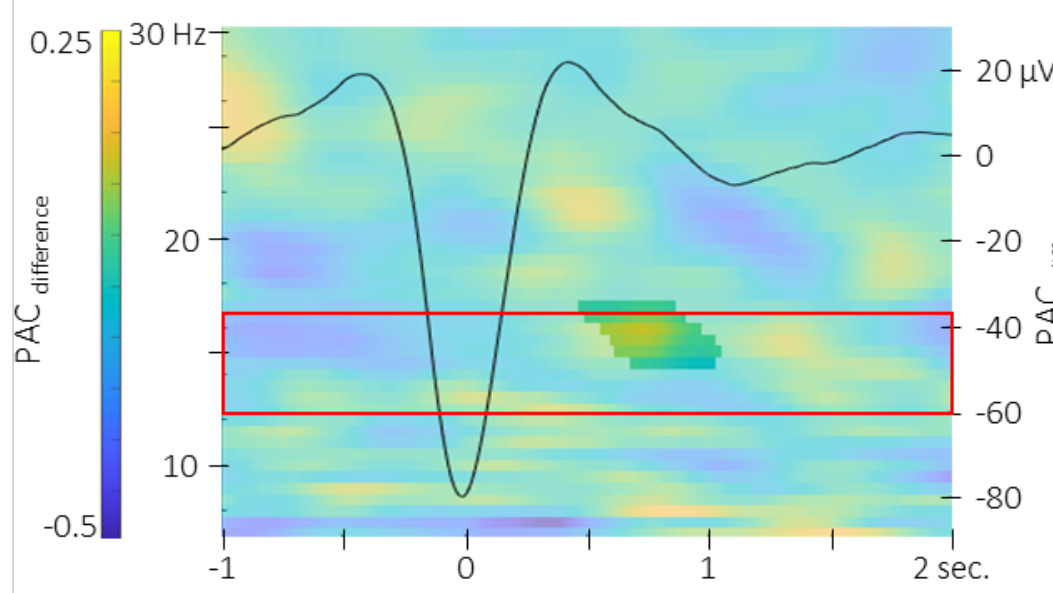

b. Correlation between PAC difference and TMR index

Figure 8: Correlation between SW-locked phase-amplitude coupling difference and TMR Index. a. Time-Frequency Representation (TFR) of the difference between the SW-locked PAC during the associated vs. unassociated stimulation intervals. Highlighted, the positive cluster in which the TMR index is significantly correlated with the difference in SWlocked PAC. Superimposed on the TFR (black line): SW grand average across individuals and conditions. Red frame highlights the pre-registered sigma frequency band of interest. $b$. Positive correlation between the SW-locked PAC difference (0.68-0.82 sec post negative peak, $14.5-17 \mathrm{~Hz}$ ) and the TMR index (dots represent individual datapoints). 


\section{Discussion}

In the present study, we examined the impact of auditory TMR on motor memory consolidation as well as the neurophysiological processes supporting reactivation during sleep. Our results demonstrate a TMRinduced behavioral advantage such that offline gains in performance were larger on the reactivated as compared to the non-reactivated sequence. These behavioral results are in line with earlier motor learning studies showing improvement in performance after auditory $(13,16,12)$ or olfactory $(17)$ TMR during sleep. Interestingly, our results show that the TMR-induced behavioral advantage was already observed in the same-day retest that immediately followed the post-learning nap and was maintained over the nocturnal sleep interval. Our findings therefore suggest that the TMR episode during a nap immediately following learning set the reactivated memory trace on a distinct yet parallel trajectory as compared to the non-reactivated memory trace.

TMR effects were also observed at the brain level such that electrophysiological responses differed according to whether they were evoked by associated or unassociated cues. Specifically, the amplitude of the negative component of the auditory ERP was higher for the sounds associated to the motor memory task as compared to the unassociated sounds. These results are in line with findings from earlier associative learning studies performed during wakefulness showing that auditory cues evoke larger responses after conditioning (i.e., after they are associated to another stimulus) and that ERP amplitude is restored to preassociation levels after extinction (see 27 for a review). The current findings also extend prior observation of a modulation of auditory-TMR-evoked responses during sleep (11). This earlier study showed that auditory cues presented during post-learning sleep evoked larger ERPs when they were associated to items better remembered at subsequent recall as compared to cues associated to less remembered items. Our findings not only concur with this post-hoc analysis, but also provide the first direct evidence of an ERP modulation based on the memory content of the cue during post-learning sleep. This difference in brain potentials during sleep might be seen as the neural signature of the plasticity processes that occurred during learning. Not exclusive to the previous speculation, such effects might also be attributed to the (re)processing of the memory trace during post-learning sleep. Importantly, one could argue that the difference in ERP amplitude observed in the present study might be due to familiarity effects, as the unassociated sound might have been perceived as novel as compared to the associated sound. We argue that this is unlikely as new or rare auditory stimuli usually present larger negative amplitudes as compared to old or frequent sounds during both sleep and wakefulness (e.g., FN $400(28,29)$ for old/new paradigms during wake and mismatch negativity components for oddball paradigms during both wake (30) and sleep $(30,31))$. Instead, we propose that the auditory evoked brain responses observed in the current study reflect the (re)processing of the motor memory trace that was encoded during initial learning.

In addition to the modulation of auditory-evoked responses described above, the properties of the detected (spontaneous) SWs were influenced by sound presentation and sound condition. Specifically, SW density was higher during sound presentation as compared to rest and the density and amplitude of the SWs were greater during intervals of associated - as compared to unassociated - cue stimulation. The effect of sound presentation on SW characteristics is in line with previous work showing sound-related entrainment of SW trains and increase in SW amplitude during sleep $(32,6,33,8)$. More importantly, in line with the ERP results, our data show that the memory content of the cue modulated SW physiology above and beyond the mere effect of sound presentation. This is the first evidence, to the best of our knowledge, of a modulation of SW physiology based on the relevance of the sensory cues presented during sleep. We speculate that the processing of the memory content associated to the cue resulted in enhanced SW activity. Specifically, the greater amplitude of the SWs during associated sound intervals might reflect neural synchronization $(34,35)$ known to promote sleep-dependent plasticity processes e.g. $(15,6)$. We thus propose that the TMR-effect observed in this study might therefore be mediated by SWs likely in relation with spindle activity. 
While the characteristics of spontaneous spindles (amplitude and frequency) were only modulated by sound presentation and not sound condition, the properties of sigma oscillations (i.e., its amplitude and its coupling with the SO phase) were differently affected by the cue type and related to the TMR-induced behavioral advantage. The observation of a modulation of spindle characteristics irrespective of the sound condition suggests that spindle activity (in term of events) during reactivation is not related to motor memory processing per se. This stands in contrast with earlier reports of spindle-mediated effect of TMR on the consolidation of both declarative e.g. $(18)$ and motor $(17,12)$ memory tasks. It is worth noting, however, that this earlier work did not compare different stimulus conditions as in the present study. Nonetheless, this previous research demonstrated that reactivation was related to an increase in spindle features (amplitude and frequency) that were linked to the TMR-induced motor performance advantage (17).

Importantly, it is worth explicitly stating that our results do not rule out the involvement of spindle activity in TMR-related motor memory consolidation processes. Recent evidence has brought forward the idea that spindle event detection in general is less sensitive than the study of the sigma rhythm as a whole (36). In line with these observations, our results show that sigma oscillation properties - as opposed to spindle events - were modulated by the sound condition and that such modulation was related to the TMRinduced behavioral advantage. Specifically, higher coupling between sigma oscillation amplitude and the SO phase, for associated as compared to unassociated sounds, on the descending phase following the peak of the SW was correlated with the TMR index. To the best of our knowledge, this is the first time that the strength of the coupling between the SO phase and the amplitude of the sigma oscillations nested within the peak of the SW is directly related to a TMR-related behavioral advantage. Earlier studies comparing different age groups provided convincing, yet indirect, evidence that the precise temporal coordination of SO and sleep spindles represents a critical mechanism for sleep-dependent memory consolidation $(10,37$, 38). The timing reported in this earlier work is consistent with the current data showing increased SWsigma coupling on the descending phase following the peak of the SW. Our results are also in line with previous frameworks proposing that sigma oscillation (18) / spindles (39) offer a privileged time window for relevant memories to be reinstated during sleep. Together with evidence that TMR boosted SW features, the current data suggest that both SWs and sigma oscillations play a critical role in the reactivation of motor memories.

In addition to the modulation of neurophysiological responses described above and triggered by the associated sounds, we report an intriguing pattern of brain results for the unassociated sounds. Specifically, the coupling between sigma amplitude and the SO phase was strengthened for unassociated sounds just before the onset of the SW negative peak. Furthermore, we observed that the increase in sigma power nested in the trough of the auditory evoked potential for unassociated (as compared to associated) sounds was related to higher TMR-induced performance enhancement. It is tempting to speculate that sigma oscillations might prevent the processing of unassociated/irrelevant sounds during post-learning sleep which might in turn be reflected by a decrease in the amplitude of the slow electrophysiological responses (i.e., smaller ERP and SWs) during non-associated sound intervals. We argue that sigma oscillations might play the role of a gatekeeper for the consolidation process and protect the motor memory trace against potential interfering effects induced by the unassociated sounds which might in turn potentiate the effect of TMR at the behavioral level. These assumptions are in line with a growing body of literature pointing towards a sensory gating role of spindle activity / sigma oscillations $(40,41)$ that might be critical to facilitate the memory consolidation process during sleep $(42,39)$. Specifically, it has been proposed that a function of the thalamus is to suppress distraction and gate information processing via alpha/beta oscillations during wakefulness (43) and sigma oscillations during sleep (44). Further support for the gating hypothesis comes from observations of both increased arousal threshold as well as decreased amplitude of auditory ERP when sounds are presented simultaneously to a spindle event (45, 46). Along the same lines, previous studies using simultaneous EEG-fMRI recordings showed that the BOLD 
responses in relation to sound processing are inconsistent or even absent when sounds occur during sleep spindles or before the negative peak of the SW $(40,41)$. The present data therefore suggest that the precise SO-sigma coordination does not only play a role in the reinstatement of relevant memories, but is also critical to prevent the processing of irrelevant information during post-learning sleep. These observations are remarkably in line with recent theoretical views putting forward the concept that temporally precise SO-spindle coupling might not convey only memory-specific information (47). It is argued that while synchronized states might trigger memory reactivation, the underlying neural activity might offer limited opportunities for information processing. Our data concur with this theory as they suggest that SO-sigma coupling, depending on its temporal characteristics, either prevents the processing of irrelevant information or supports memory reactivation during post-learning sleep.

In conclusion, our results depict a complex organization of the different physiological processes supporting motor memory consolidation during post-learning sleep. While associated sounds appeared to boost SW features and SW-sigma coupling at the peak of the SW, unassociated sounds predominantly modulated the properties of the sigma oscillations at the trough of the slow oscillation. Our findings suggest a dual role of sigma oscillations whereby, depending on their temporal coordination with SWs, they either protect memories against irrelevant material processing or promote the reactivation of relevant motor memories; two processes that critically contribute to the motor memory consolidation process. 


\section{Materials and Methods}

This study was pre-registered in the Open Science Framework (https://osf.io/). Our pre-registration document outlined our hypotheses and intended analysis plan as well as the statistical models used to test our a priori hypotheses (available at osf.io/n3me8). Whenever an analysis presented in the current paper was not pre-registered, it is referred to as exploratory. Additionally, in order to increase transparency, any deviation from the pre-registration is marked with a (\#) symbol and listed in supplemental Table S1 together with a justification for the change.

\subsection{Participants}

Young healthy volunteers were recruited by local advertisements to participate in the present study. Participants gave written informed consent before participating in this research protocol, approved by the local Ethics Committee (B322201525025) and conducted according to the declaration of Helsinki (2013). The participants received a monetary compensation for their time and effort. Inclusion criteria were: 1) left- or right-handed\# (see supplemental Table S1.1); 2) no previous extensive training with a musical instrument or as a professional typist, 3) free of medical, neurological, psychological, or psychiatric conditions, including depression and anxiety as assessed by the Beck's Depression (48) and Anxiety (49) Inventories, 4) no indications of abnormal sleep, as assessed by the Pittsburgh Sleep Quality Index (50); 5) not considered extreme morning or evening types, as quantified with the Horne \& Ostberg chronotype questionnaire (51); and, 6) free of psychoactive or sleep-affecting medications. None of the participants were shift-workers or took trans-meridian trips in the 3 months prior to participation.

The sample size was determined with a power analysis performed through the G*Power software (52) and based on the paper of Cousins et al. (12) which reports, to our knowledge, the closest paradigm to the present one in the motor memory domain (see supplemental material \& methods).

Thirty-four participants took part in the study to reach the estimated sample size after participant exclusion. As per our pre-registration, participants were excluded if their sleep duration during the experimental nap was insufficient to provide at least 50 stimulations per condition (after EEG data cleansing). This cut-off aimed at providing enough events to reach sufficient signal-to-noise ratio for electrophysiological analyses. Ten participants did not reach this criterion; accordingly, 24 participants (12 females) completed the experimental protocol and were included in the analyses (see participants' characteristics in Table 3).

Table 3. Participant characteristics

\begin{tabular}{ll}
\hline $\mathrm{N}$ & 24 (12 females) \\
Age (yrs) & 21.9 ranging from 18 to 27 \\
Edinburgh Handedness (53) & $78.6[57.1-100]$ \\
Epworth Sleepiness Scale (54) & $7[5.9-8.1]$ \\
Beck Depression Scale (48) & $1.5[0.9-2.2]$ \\
Beck Anxiety Scale (49) & $1.8[1.1-2.4]$ \\
PSQI (50) & $3[2.2-3.8]$ \\
Chronoscore (CRQ)(51) & $48.8[45.6-51.9]$ \\
\hline
\end{tabular}

Notes. Values are means [lower and upper limit of the 95\% Confidence Interval - CI]. PSQI = Pittsburgh Sleep Quality Index; $C R Q$ = Circadian Rhythm Questionnaire.

\subsection{General design}

The study design was a within-participant design (Figure 1). Participants were first invited, in the early afternoon, for a habituation nap during which they completed a 90-minute nap monitored with polysomnography (PSG, see below for details). Approximately one week later, participants returned to complete the experimental protocol. Each participant followed a constant sleep/wake schedule (according 
to their own rhythm $+/-1 \mathrm{~h}$ ) for the 3 days before the experiment. Compliance was assessed with sleep diaries and wrist actigraphy (ActiGraph wGT3X-BT, Pensacola, FL). Sleep quality and quantity for the night preceding the experimental visit was assessed with the St. Mary's sleep questionnaire (55) (see supplemental results for sleep data before the experimental session). During the first experimental day, participants were trained on two motor sequences simultaneously (pre-nap session: between 12 pm-1:30 $\mathrm{pm}$ ). During learning, each of these two sequences was associated to a particular sound. Only one of these 2 sounds was presented during the subsequent nap episode, corresponding to the associated sound linked to the reactivated sequence. At the behavioral level, the control condition consisted of the non-reactivated sequence (i.e., a sequence that was associated to a sound during learning but the sound was not presented during the subsequent nap interval). For electrophysiological analyses, a new, unassociated, sound (i.e., a sound to which participants were not exposed during the learning episode) was presented during the postlearning sleep, serving as a control condition. The nap occurred between 1:30 pm and $3 \mathrm{pm}$ and was monitored with PSG. Sleep data were monitored online by an experimenter in order to send auditory stimulations during NREM2-3 stages. Performance on the reactivated and non-reactivated sequences was tested 30 min after the end of the nap to allow sleep inertia to dissipate (post-nap session: 2 pm-5:30 pm) and on day 2 after a night of sleep (not monitored with PSG) spent at home (post-night session: 8:30 am11:30 am). At the beginning of each behavioral session, vigilance was measured objectively and subjectively using the Psychomotor Vigilance Task (56) and Stanford Sleepiness Scale (54), respectively (see supplemental document). Finally, general motor execution was tested at the beginning of the prenap session and at the end of the post-night session (see supplemental document).

This design allowed to assess the specific impact of TMR on consolidation at the behavioral level, with the comparison between the performance gains of the reactivated and non-reactivated sequences assessed during the post-nap and post-night sessions; and at the electrophysiological level, with the comparison between the neurophysiological responses to the reactivated associated sound vs. the unassociated sound that did not carry mnemonic information.

\subsection{Stimuli and tasks}

All tasks were performed on a laptop computer (Dell Latitude 5490 run under Microsoft Windows 10 Enterprise) and were implemented in Matlab (Math Works Inc., Natick, MA, USA) Psychophysics Toolbox version 3 (57). Participants sat comfortably in front of the computer screen with the keyboard on their knees. This configuration allowed the participants to focus their gaze on the screen and not to look at their hands/movements. Distance between participants and the screen was approximately $70 \mathrm{~cm}$ but was selfselected by the participants based on comfort. The sound presentation was conducted using ER3C air tube insert earphones (Etymotic Research).

\subsubsection{Acoustic stimulation}

Three different 100-ms sounds were randomly assigned to the three conditions (reactivated/associated, not-reactivated, and unassociated), for each participant. The three synthesized sounds consisted of a tonal harmonic complex created by summing a sinusoidal wave with a fundamental frequency of $543 \mathrm{~Hz}$ and 11 harmonics with linearly decreasing amplitude (i.e. the amplitude of successive harmonics is multiplied by values spaced evenly between 1 and 0.1); white noise band-passed between 100-1000 Hz and a tonal harmonic complex created with a fundamental frequency of $1480 \mathrm{~Hz}$ and 11 harmonics with linearly increasing amplitude (i.e. the amplitude of successive harmonics is multiplied by values spaced evenly between 0.1 and 1). A 10-ms linear ramp was applied to the onset and offset of the sound files so as to avoid earphone clicks. At the start of the experiment, auditory detection thresholds were determined by the participants themselves using a transformed 1-down 1-up procedure $(57,58)$ separately for each of 
the three sounds. Subsequently, the sound pressure level was set to $1000 \%$ of the individual auditory threshold during the tasks and $140 \%$ for auditory stimulation during sleep, thus limiting the risk of awakening during the nap (60). Before the start of the nap episode, participants were instructed that they may or may not receive auditory stimulations during the nap.

\subsubsection{Motor Task}

A bimanual serial reaction time task (22) (SRTT) was used to probe motor learning and memory consolidation processes. During this task, eight squares were horizontally presented on the screen meridian, each corresponding to one of the eight keys on the specialized keyboard and to one of the 8 fingers (no thumbs). The color of the outline of the squares alternated between red and green, indicating rest and practice blocks, respectively. During the practice blocks, participants had to press as quickly as possible the key corresponding to the location of a green filled square that appeared on the screen. After a response, the next square changed to green with a response-to-stimulus interval of $0 \mathrm{~ms}$. After 64 presses, the practice block automatically turned into a rest block and the outline of the squares changed from green to red. The rest interval was $15 \mathrm{sec}$.

The order in which the squares were filled green (and thus the order of the key presses) either followed a sequential or pseudo-random pattern. In the sequential SRTT, i.e. assessing motor sequence learning, participants were trained simultaneously on two different eight-element sequences (sequence A: 1635 482 7; sequence B: 7264518 3, in which 1 through 8 are the left pinky to the right pinky fingers respectively). Participants were explicitly told that the stimuli (and thus the finger presses) would follow two different repeating patterns composed of eight elements each, but were not told any further information. During each practice block, four repetitions of a specific sequence (e.g. sequence A) were performed, each separated by a 1 sec-interval. Then, after a 2 sec-interval, the four repetitions of the other sequence started (e.g. sequence B). The order of the two sequences was randomized within each block of practice. Each motor sequence was associated to a different tone that consisted of a single 100-ms auditory cue (see above). The auditory cue was presented before the beginning of each sequence repetition, i.e. before the first key press of the sequence that was to be performed. Accordingly, one single tone was associated to an eight-element sequence of finger movements. Participants were instructed to learn the sequence-sound association during task practice. The associations between sound-sequence (sounds 1, 2 and 3; sequence $A$, sequence $B$, and control sound presented during nap) and sequencecondition (sequences $A$ and $B$; conditions reactivated and not-reactivated) were randomized, thus creating 12 different possible combinations of randomized variables. Each participant was pseudo-randomly assigned to one of these combinations, such that there were two participants per combination. For the random SRTT, the order of the eight keys was shuffled for each eight-element repetition and thus the number of each key press was constant across all random and sequential blocks. For both variants of the task, the participants were instructed to focus on both speed and accuracy.

For the pre-nap session, participants first completed 4 blocks of the random SRTT to assess general motor execution. Participants subsequently completed the sequential SRTT, which consisted of 16 blocks of training followed by 4 blocks of post-training test taking place after a 5 -min break (24). This allowed the assessment of end of training performance after the further dissipation of physical and mental fatigue (24). Between the training and test runs, participants completed a generation task that aimed at testing participants' explicit knowledge of the sequences as well as the strength of the association between the sequences and their corresponding auditory cues (see supplemental document). For the post-nap session, only 4 blocks of the sequential SRTT were completed to avoid extensive task practice before the final 
overnight retest. For the post-night session, 16 blocks of the sequential SRTT were performed, followed by the generation assessment and finally 4 blocks of the random SRTT.

Indexes reflecting sequence-specific knowledge and sequence awareness were computed using random and generation data, respectively. Corresponding methods and results are reported in the supplemental document.

\subsection{Polysomnography and Targeted Memory Reactivation protocol}

Both habituation and experimental naps were monitored with a digital sleep recorder ( $\mathrm{V}$-Amp, Brain Products, Gilching, Germany; bandwidth: DC to Nyquist frequency) and were digitized at a sampling rate of $1000 \mathrm{~Hz}$ (except for one participant $(500 \mathrm{~Hz})$ due to experimental error). Standard electroencephalographic (EEG) recordings were made from $\mathrm{Fz}, \mathrm{C} 3, \mathrm{Cz}, \mathrm{C} 4, \mathrm{Pz}, \mathrm{Oz}, \mathrm{A} 1$ and $\mathrm{A} 2$ according to the international $10-20$ system (note that $\mathrm{Fz}, \mathrm{Pz}$ and $\mathrm{Oz}$ were omitted during the habituation nap). A2 was used as the recording reference and A1 as a supplemental individual EEG channel. An electrode placed on the middle of the forehead was used as the recording ground. Bipolar vertical and horizontal eye movements (electrooculogram: EOG) were recorded from electrodes placed above and below the right eye and on the outer canthus of both eyes, respectively. Bipolar submental electromyogram (EMG) recordings were made from the chin. Electrical noise was filtered using a $50 \mathrm{~Hz}$ notch. Impedance was kept below $5 \mathrm{k} \Omega$ for all electrodes. During the experimental nap, PSG recordings were monitored by a researcher in order to detect NREM2-3 sleep based on the most recent sleep scoring guidelines from the American Academy of Sleep Medicine (61). To do so, PSG recordings were displayed online using 30-second-long epochs with EEG and EOG data filtered from 0.5 to $30 \mathrm{~Hz}$ and EMG data filtered between 20-200 Hz. When NREM2-3 sleep stages were reached, auditory cues were sent. The auditory stimulation was presented in a blocked design (Figure 1B). Namely, each type of auditory cue (associated or unassociated) was sent during 3-minute-long stimulation intervals with an inter-stimulus interval of $5 \mathrm{sec}$. The stimulation was stopped manually when the experimenter detected REM sleep, NREM1 or wakefulness. Intervals of stimulation for each sound were separated by a 1-minute silent period (rest intervals).

\subsection{Analysis}

Statistical tests were performed with the open-source software R (62) (63) and considered significant for $p<0.05$. When necessary, corrections for multiple comparisons was conducted with the False Discovery Rate(64) (FDR) procedure within each family of hypothesis tests (see details for each analysis below). Greenhouse-Geisser corrections was applied in the event of the violation of sphericity. Wilcoxon signedrank tests were used when the Shapiro-Wilk test indicated non-normal distribution ${ }^{\#}$ (see supplemental Table S1.6). $F, t$ and $V$ (or $W$ ) statistics and corrected $p$-values were therefore reported for ANOVAs, student and Wilcoxon tests, respectively. Effect sizes are reported for significant comparisons using Cohen's $d$ for Student t-tests, $r$ for Wilcoxon signed-rank test and $\eta^{2}$ for rmANOVAs using G*power (52). For correlation analyses, Spearman\# test (see supplemental Table S1.6) was used and S as well as corrected p-values were reported. Nonparametric CBP tests (25) implemented in fieldtrip toolbox(65) were used for high dimensional time and time-frequency data analyses (e.g. ERP, TF and PAC analyses). CBP tests are composed of two subsequent tests. The first calculates paired t-tests (for contrast analyses) between conditions for each time points (or time-frequency points), which are then thresholded at a chosen p-value which sets the conservativeness of the test (reported as "cluster threshold"). Significant clusters are defined as showing a continuum of significant time (or time-frequency) points. Subsequently, the procedure is repeated 500 times on shuffled data in which the condition assignment within each individual 
is permuted randomly. On each permutation, the maximum t-value is retained, yielding a distribution of $500 \mathrm{t}$-values (for contrast analyses). Finally, this distribution is used as a reference to determine whether the statistical value ( $t$ in case of contrast analyses) of each cluster, as calculated on the real assignment of the conditions, is likely to come from the same probability distribution ( $p$-value $>0.05$ ) or rather differs significantly from this random perturbation probability distribution ( $p$-value $<0.05$ ). For CBP contrast analyses, Cohen's $d$ is reported while rho is reported for CBP correlations.

\subsubsection{Behavior}

\subsubsection{Preprocessing}

Motor performance on both the random and sequential SRTT was measured in terms of speed (correct response time RT in ms) and accuracy (\% correct responses) for each block of practice. Note that RTs from individual correct trials were excluded from the analyses if they were greater than 3 standard deviations above or below the participant's mean correct response time for that block ( $1.73 \%$ in total). Consistent with our pre-registration, our primary analyses were performed on speed. For completeness, accuracy analyses are presented in the supplemental material.

The offline performance gains on the sequential SRTT were computed as the relative change in speed between the pre-nap session (namely the 3 last blocks of practice", see results and supplemental Table 1.2 for details) and the post-nap session (4 blocks of practice) and the post-night session (4 first blocks of practice) separately for the reactivated and the non-reactivated sequences. A positive offline gain in performance therefore reflects an increase of absolute performance from the pre-nap test to the postnap or post-night tests. Additionally, we computed a TMR index, to be used in brain-behavior correlation analyses, which consisted of the difference in offline gain in performance between the reactivated and non-reactivated sequences (being positive when reactivated offline gain is higher than non-reactivated one).

\subsubsection{Statistical analyses}

We first assessed whether performance significantly differ between conditions during initial training. To do so, two two-way rmANOVAs with Condition (reactivated vs. non-reactivated) and Block ( $1^{\text {st }}$ rmANOVA on the 16 blocks of the pre-nap training and $2^{\text {nd }}$ rmANOVA on the 4 blocks of the pre-nap test) as withinsubject factors were performed on the sequential SSRT performance. Similar analyses testing for baseline differences between sequences $A$ and $B$ irrespective of the reactivation condition were performed (see supplemental results). We then tested whether offline gains in performance on the sequential SRTT differed between reactivation conditions after a nap and night of sleep. This was done with a rmANOVA with Time-point (post-nap vs. post-night) and Condition (reactivated vs. non-reactivated) as within-subject factors on the offline performance gains.

\subsubsection{Electroencephalography}

\subsubsection{Offline sleep scoring}

A certified sleep technologist blind to the stimulation periods completed the sleep stage scoring offline according to criteria defined in (66) using the software SleepWorks (version 9.1.0 Build 3042, Natus Medical Incorporated, Ontario, Canada). Data were visually scored in 30-second epochs and band pass filters were applied between 0.3 and $35 \mathrm{~Hz}$ for EEG signals, 0.3 and $30 \mathrm{~Hz}$ for EOG, and 10 and $100 \mathrm{~Hz}$ for EMG. A $50 \mathrm{~Hz}$ notch filter was also used (see Table 1 for details of extraction from scored data). 


\subsubsection{Preprocessing}

EEG data preprocessing was carried out using functions supplied by the fieldtrip toolbox (65). Specifically, data were cleaned by manually screening each 30-sec epoch. Data segments contaminated with muscular activity or eye movements were excluded. Data were filtered between 0.1-30 Hz.

\subsubsection{Event-related analyses}

Event-related data analyses (i.e., auditory-evoked potentials and oscillatory activity) were performed with the fieldtrip toolbox (65) with down sampled data $(100 \mathrm{~Hz})$. Auditory-evoked responses were obtained by segmenting the data into epochs time-locked to auditory cue onset (from - 1 to 3 sec relative to auditory cue onset after correction for onset-trigger lags) separately for the associated and unassociated auditory cues and averaged across all trials\# (see supplemental Table 1.3) in each condition separately. During cleaning, $1.03 \%$ [ $95 \% \mathrm{Cl}: 0.49-1.58$ ] of the trials with stimuli sent during NREM2-3 stages were discarded. The remaining number of artifact-free trials was not significantly different between the two stimulation conditions (associated vs. unassociated, $t=-0.5888, d f=23, p$-value $=0.5617$ ).

For event-related potentials (ERPS) analyses, individual ERPS were baseline corrected by subtracting mean amplitude from -0.3 to $-0.1 \mathrm{sec}$ relative to cue onset. All 6 EEG channels were averaged together. To identify specific time windows to compare ERP amplitude between conditions, we used CBP approaches on ERP data computed across conditions. Results showed that across condition ERP was significantly different from zero between $0.44-0.63 \mathrm{sec}$ at the trough (alpha threshold $=0.025$, cluster $\mathrm{p}$-value $=0.044$; Cohen's $d=-0.56$ ) (see supplemental Figure S2). ERP amplitude was then averaged within this specific time-window for the 2 conditions separately and compared using one-tailed paired Wilcoxon signed-rank test\# (see supplemental Table S1.6) with the hypothesis that ERP absolute amplitude at the trough is greater following the associated cues as compared to unassociated cues.

To analyze oscillatory activity, we computed Time-Frequency Representations (TFRs) of the power spectra per experimental condition. To this end, we used an adaptive sliding time window of five cycles length per frequency ( $\Delta t=5 / f ; 20$-ms step size), and estimated power using the Hanning taper/FFT approach between 5 and $30 \mathrm{~Hz}^{\#}$ (see supplemental Table 1.4). Individual TFRs were converted into baseline relative change of power (baseline from -0.3 to $-0.1 \mathrm{sec}$ relative to cue onset), thus highlighting power modulation following the auditory cues. All 6 EEG channels were then averaged. TFR locked to associated vs. unassociated auditory cues were compared using a CBP test between 5 to $30 \mathrm{~Hz}$ and from 0 to $2.5 \mathrm{sec}$ relative to cue onset.

\subsubsection{Sleep-event detection}

Preprocessed cleaned data were down-sampled to $500 \mathrm{~Hz}$ and were transferred to the python environment. Slow waves and spindles were detected automatically in NREM2-3 sleep epochs on all the channels, by using algorithms implemented in the YASA open-source Python toolbox (26). Events were defined as SWs if they met the following criteria adapted from (67): a frequency comprised between 0.5 and $2 \mathrm{~Hz}$, duration of the negative peak between 0.3 and $1.5 \mathrm{sec}$, duration of the positive peak between 0.1 and $1 \mathrm{sec}$, amplitude of the negative peak between 40 and $300 \mu \mathrm{V}$, amplitude of the positive peak between 10 and $100 \mu \mathrm{V}$ and PTP amplitude between 75 and $500 \mu \mathrm{V}$. Events were defined as spindles if they met the following criteria adapted from (68): a duration comprised between 0.5 and $3 \mathrm{sec}$ and a frequency between 12 and $16 \mathrm{~Hz}$.

SWs and spindles were detected in the stimulation intervals of both associated and unassociated sounds. One participant did not show any SW during the unassociated cue stimulation intervals and the minimal required number of SWs was not reached to perform the PAC in another participant. The two participants were thus excluded from the analyses on detected SWs. No spindles were detected during the associated 
cue stimulation intervals for another participant who was therefore excluded from the spindle analyses. With respect to the detected SWs, we extracted for each participant and condition, the mean PTP amplitude $(\mu \mathrm{V})$ of SW $\mathrm{s}^{\#}$ (see supplemental Table S1.3) as well as their density (number of SWs per total time in minutes spent in stimulation or rest intervals). Concerning the spindles, we extracted for each participant and condition spindle density (i.e., the number of spindles per total time in minutes spent in stimulation or rest intervals). Spindle amplitude (computed as the PTP amplitude $(\mu \mathrm{V})$ in the non-filtered data) and frequency were also extracted for exploratory analyses. These different dependent variables were compared using a one-tailed paired Student t-test (SW PTP and spindle Frequency) or Wilcoxon signed-rank (SW density, spindle density and amplitude) test\# (see supplemental Table S1.6) with the hypothesis that the associated, as compared to unassociated, stimulation intervals would exhibit higher values.

Furthermore, we performed exploratory analyses including the SWs and the spindles detected during rest intervals (i.e. NREM 2-3 epochs without auditory stimulation). In the case of SWs, we compared these values with those obtained for the associated stimulation intervals and the unassociated stimulation intervals using two two-tailed Student t-tests or Wilcoxon signed-rank tests (rest vs. associated stimulation intervals and rest vs. unassociated stimulation intervals). In the case of spindles, as spindle characteristics did not differ between stimulation conditions (see results), they were collapsed across stimulation conditions and compared to rest intervals using two-tailed Student t-tests or Wilcoxon signed-rank tests. Correction for multiple comparisons was performed using the FDR approach (64).

\subsubsection{Phase-amplitude coupling}

Preprocessed cleaned data were down-sampled to $500 \mathrm{~Hz}$, all 6 EEG channels were averaged together, and were transferred to the python environment. We tested, using the tensorPac (69) open-source Python toolbox, whether and when the phase of the $0.5-2 \mathrm{~Hz}$ oscillatory signal was coupled with the amplitude of the signal in the 7-30 Hz frequency range ${ }^{\#}$ (see supplemental Table S1.4) in relation with either the auditory cue onset or the negative peak of the SWs. To do so, the raw data were filtered between $0.5-2 \mathrm{~Hz}$ and 7 $30 \mathrm{~Hz}$. Next, the complex analytical form of each signal was obtained using the Hilbert transform. The phase of the signal from -0.5 to $2.5 \mathrm{sec}$ around the auditory cue onset and signal from - 1 to $2 \mathrm{sec}$ around the negative peak of the SWs was extracted from the filtered signal within the $0.5-2 \mathrm{~Hz}$ SO frequency band. The amplitude of the signal was also computed in the windows described above between 7 and $30 \mathrm{~Hz}$ with $0.5 \mathrm{~Hz}$ step size. The strength of the coupling between the phase of the SO signal and the amplitude of the 7-30 Hz signal was then computed at each time point of the analysis window (i.e., every $2 \mathrm{~ms}$ ) separately for the two sound conditions and compared using CBP test. Additionally, we performed exploratory analyses in which PAC (computed relative to the negative peak of the SWs as described above) was extracted from rest intervals. We compared rest PAC to PAC derived from both the associated and unassociated stimulation intervals using CBP procedures and corrected for two comparisons using the FDR.

The preferred phase (PP), which reflects whether the amplitude of the signal in a given frequency band is modulated by the phase of the signal in another band, was also computed using tensorPac (69) opensource Python toolbox. Based on our a priori hypotheses, these analyses focused on the amplitude of the signal in the sigma band and the phase of the SO. The amplitude was binned according to phase slices. The preferred phase is given by the phase bin at which the amplitude is maximum. The PP was compared between the two stimulation conditions using a Watson test for circular data\# (see supplemental Table S1.5) a critical value for 24 participants of 0.187 with alpha level $=0.05$. Similar as above, PP was also extracted from rest intervals for exploratory analyses in which rest PP was compared to the PP derived from the two different stimulation intervals using Watson test for circular data.

\subsubsection{Correlational analyses}


Following our pre-registration, we performed correlation analyses between the TMR index and the following EEG-derived data: (1) The difference between the densities of SWs detected during the associated and unassociated cue stimulation intervals using one-sided Spearman\# ${ }^{\#}$ correlations (supplemental Table S1.6); (2) The difference between the densities of spindles detected during the associated and unassociated cue stimulation intervals using one-sided Spearman\# correlations; (3) The relative change between the amplitude of the negative peak of the ERP\# (supplemental Table S1.3) following the associated and unassociated auditory cues using one-sided Spearman ${ }^{\#}$ correlations; (4) The difference in auditory-locked sigma band power (0-2.5 sec relative to cue onset and from 12 to $16 \mathrm{~Hz})$ between the associated and unassociated auditory cues using CBP tests ${ }^{\#}$ (supplemental Table S1.7); (5) The difference between SO phase and sigma oscillation amplitude $(12-16 \mathrm{~Hz})$ coupling strength during the associated and unassociated stimulation intervals in relation to the cue onset and to the SW event using CBP approaches\# (supplemental Table S1.6). For all one-sided tests, we predicted that the TMR index would be positively correlated with the EEG-derived metrics. 


\section{Acknowledgments}

This work was supported by the Belgian Research Foundation Flanders (FWO; G0D7918N), The Fond de Recherche en santé du Québec en sciences naturelles (RRQNT-2018-264146), Healthy Brain for Healthy Lives Discovery Grant Program from the Canada First Research Excellence Fund and internal funds from KU Leuven. GA also received support from FWO (G0B1419N, G099516N, 1524218N) and Excellence of Science (EOS, 30446199, MEMODYN, with SS). Financial support for authors JN and BRK was provided by the European Union's Horizon 2020 research and innovation program under the Marie Skłodowska-Curie grant agreement (\#887955 and \#703490, respectively). Finally, we would like to thank dr. Raphaël Vallat for his valuable help when author JN first approached YASA open-source Python toolbox.

\section{Author Contributions}

BK, GA, JD, JC and SS initiated the bilateral FWO-FRQ research project. JN, GA, and BK designed the study. EC provided help on the design of the auditory stimuli. DL and LL provided insight on the data analysis. JN acquired the data, analyzed the data and wrote the first draft of the manuscript. BK and GA provided extensive feedback. DL, LL, EC, SS, JD, and JC reviewed and edited the manuscript.

\section{Declaration of Interests}

Dr. Carrier serves as a consultant for Eisai and Haleo.

\section{Data availability}

Data will be made publicly available upon publication.

\section{Code availability}

All custom scripts can be accessed upon request reasonable request.

\section{References}

1. Maquet P. The Role of Sleep in Learning and Memory. Science. 2 nov 2001;294(5544):1048-52.

2. Robertson EM, Pascual-Leone A, Miall RC. Current concepts in procedural consolidation. Nat Rev Neurosci. juill 2004;5(7):576-82.

3. Albouy $G$, Sterpenich V, Balteau E, Vandewalle $G$, Desseilles $M$, Dang-Vu $T$, et al. Both the Hippocampus and Striatum Are Involved in Consolidation of Motor Sequence Memory. Neuron. avr 2008;58(2):261-72.

4. Albouy G, Fogel S, Pottiez H, Nguyen VA, Ray L, Lungu O, et al. Daytime Sleep Enhances Consolidation of the Spatial but Not Motoric Representation of Motor Sequence Memory. van Swinderen B, éditeur. PLoS ONE. 2 janv 2013;8(1):e52805.

5. King BR, Hoedlmoser K, Hirschauer F, Dolfen N, Albouy G. Sleeping on the motor engram: The multifaceted nature of sleep-related motor memory consolidation. Neurosci Biobehav Rev. sept 2017;80:1-22.

6. Ngo H-VV, Martinetz T, Born J, Mölle M. Auditory Closed-Loop Stimulation of the Sleep Slow Oscillation Enhances Memory. Neuron. mai 2013;78(3):545-53. 
7. Barakat M, Carrier J, Debas K, Lungu O, Fogel S, Vandewalle G, et al. Sleep spindles predict neural and behavioral changes in motor sequence consolidation: Sleep Spindles Predict Motor Consolidation. Hum Brain Mapp. nov 2013;34(11):2918-28.

8. Ngo H-VV, Seibold M, Boche DC, Mölle M, Born J. Insights on auditory closed-loop stimulation targeting sleep spindles in slow oscillation up-states. J Neurosci Methods. mars 2019;316:117-24.

9. Axmacher N, Elger CE, Fell J. Ripples in the medial temporal lobe are relevant for human memory consolidation. Brain. 1 juill 2008;131(7):1806-17.

10. Muehlroth BE, Sander MC, Fandakova Y, Grandy TH, Rasch B, Shing YL, et al. Precise Slow Oscillation-Spindle Coupling Promotes Memory Consolidation in Younger and Older Adults. Sci Rep. déc 2019;9(1):1940.

11. Rudoy JD, Voss JL, Westerberg CE, Paller KA. Strengthening Individual Memories by Reactivating Them During Sleep. Science. 20 nov 2009;326(5956):1079-1079.

12. Cousins JN, El-Deredy W, Parkes LM, Hennies N, Lewis PA. Cued Reactivation of Motor Learning during Sleep Leads to Overnight Changes in Functional Brain Activity and Connectivity. Battaglia FP, éditeur. PLOS Biol. 3 mai 2016;14(5):e1002451.

13. Schönauer M, Geisler T, Gais S. Strengthening Procedural Memories by Reactivation in Sleep. J Cogn Neurosci. janv 2014;26(1):143-53.

14. Hu X, Cheng LY, Chiu MH, Paller KA. Promoting memory consolidation during sleep: A metaanalysis of targeted memory reactivation. Psychol Bull. mars 2020;146(3):218-44.

15. Born J, Wilhelm I. System consolidation of memory during sleep. Psychol Res. mars 2012;76(2):192-203.

16. Antony JW, Gobel EW, O'Hare JK, Reber PJ, Paller KA. Cued memory reactivation during sleep influences skill learning. Nat Neurosci. août 2012;15(8):1114-6.

17. Laventure S, Fogel S, Lungu O, Albouy G, Sévigny-Dupont P, Vien C, et al. NREM2 and Sleep Spindles Are Instrumental to the Consolidation of Motor Sequence Memories. Battaglia FP, éditeur. PLOS Biol. 31 mars 2016;14(3):e1002429.

18. Cairney SA, Guttesen A á V, El Marj N, Staresina BP. Memory Consolidation Is Linked to SpindleMediated Information Processing during Sleep. Curr Biol. mars 2018;28(6):948-954.e4.

19. Göldi M, van Poppel EAM, Rasch B, Schreiner T. Increased neuronal signatures of targeted memory reactivation during slow-wave up states. Sci Rep. déc 2019;9(1):2715.

20. King BR, Gann MA, Mantini D, Doyon J, Albouy G. Persistence of Hippocampal and Striatal Multivoxel Patterns during Awake Rest after Motor Sequence Learning [Internet]. Neuroscience; 2021 juin [cité 12 août 2021]. Disponible sur: http://biorxiv.org/lookup/doi/10.1101/2021.06.29.450290

21. Rasch B, Buchel C, Gais S, Born J. Odor Cues During Slow-Wave Sleep Prompt Declarative Memory Consolidation. Science. 9 mars 2007;315(5817):1426-9.

22. Nissen MJ, Bullemer P. Attentional requirements of learning: Evidence from performance measures. Cognit Psychol. janv 1987;19(1):1-32. 
23. King BR, Dolfen N, Gann MA, Renard Z, Swinnen SP, Albouy G. Schema and Motor-Memory Consolidation. Psychol Sci. juill 2019;30(7):963-78.

24. Pan SC, Rickard TC. Sleep and motor learning: Is there room for consolidation? Psychol Bull. juill 2015;141(4):812-34.

25. Maris E, Oostenveld R. Nonparametric statistical testing of EEG- and MEG-data. J Neurosci Methods. août 2007;164(1):177-90.

26. Vallat R. raphaelvallat/yasa: v0.1.9 [Internet]. Zenodo; 2020 [cité 15 janv 2021]. Disponible sur: https://zenodo.org/record/3646596

27. Christoffersen GRJ, Schachtman TR. Electrophysiological CNS-processes related to associative learning in humans. Behav Brain Res. janv 2016;296:211-32.

28. Rugg MD, Curran T. Event-related potentials and recognition memory. Trends Cogn Sci. juin 2007;11(6):251-7.

29. Paller KA, Lucas HD, Voss JL. Assuming too much from 'familiar' brain potentials. Trends Cogn Sci. juin 2012;16(6):313-5.

30. Näätänen $R$, Paavilainen $P$, Rinne $T$, Alho $K$. The mismatch negativity (MMN) in basic research of central auditory processing: A review. Clin Neurophysiol. déc 2007;118(12):2544-90.

31. Ruby P, Caclin A, Boulet S, Delpuech C, Morlet D. Odd Sound Processing in the Sleeping Brain. 20(2):16.

32. Ngo H-VV, Claussen JC, Born J, Mölle M. Induction of slow oscillations by rhythmic acoustic stimulation: Acoustic stimulation during sleep. J Sleep Res. févr 2013;22(1):22-31.

33. Ngo H-VV, Miedema A, Faude I, Martinetz T, Molle M, Born J. Driving Sleep Slow Oscillations by Auditory Closed-Loop Stimulation--A Self-Limiting Process. J Neurosci. 29 avr 2015;35(17):6630-8.

34. Carrier J, Viens I, Poirier G, Robillard R, Lafortune M, Vandewalle G, et al. Sleep slow wave changes during the middle years of life: Changes in slow waves with age. Eur J Neurosci. févr 2011;33(4):758-66.

35. Esser SK, Hill SL, Tononi G. Sleep Homeostasis and Cortical Synchronization: I. Modeling the Effects of Synaptic Strength on Sleep Slow Waves. Sleep. déc 2007;30(12):1617-30.

36. Dimitrov $\mathrm{T}$, He M, Stickgold R, Prerau MJ. Sleep spindles comprise a subset of a broader class of electroencephalogram events. Sleep. 15 avr 2021;zsab099.

37. Helfrich RF, Mander BA, Jagust WJ, Knight RT, Walker MP. Old Brains Come Uncoupled in Sleep: Slow Wave-Spindle Synchrony, Brain Atrophy, and Forgetting. Neuron. janv 2018;97(1):221230.e4.

38. Hahn MA, Heib D, Schabus M, Hoedlmoser K, Helfrich RF. Slow oscillation-spindle coupling predicts enhanced memory formation from childhood to adolescence. eLife. 24 juin 2020;9:e53730.

39. Antony JW, Schönauer M, Staresina BP, Cairney SA. Sleep Spindles and Memory Reprocessing. Trends Neurosci. janv 2019;42(1):1-3. 
40. Dang-Vu TT, Bonjean M, Schabus M, Boly M, Darsaud A, Desseilles M, et al. Interplay between spontaneous and induced brain activity during human non-rapid eye movement sleep. Proc Natl Acad Sci. 13 sept 2011;108(37):15438-43.

41. Schabus M, Dang-Vu TT, Heib DPJ, Boly M, Desseilles M, Vandewalle G, et al. The Fate of Incoming Stimuli during NREM Sleep is Determined by Spindles and the Phase of the Slow Oscillation. Front Neurol [Internet]. 2012 [cité 1 juill 2021];3. Disponible sur: http://journal.frontiersin.org/article/10.3389/fneur.2012.00040/abstract

42. Schreiner T, Staudigl T. Electrophysiological signatures of memory reactivation in humans. Philos Trans R Soc B Biol Sci. 25 mai 2020;375(1799):20190293.

43. Jensen O, Mazaheri A. Shaping Functional Architecture by Oscillatory Alpha Activity: Gating by Inhibition. Front Hum Neurosci [Internet]. 2010 [cité 26 août 2016];4. Disponible sur: http://journal.frontiersin.org/article/10.3389/fnhum.2010.00186/abstract

44. Chen Z, Wimmer RD, Wilson MA, Halassa MM. Thalamic Circuit Mechanisms Link Sensory Processing in Sleep and Attention. Front Neural Circuits [Internet]. 5 janv 2016 [cité 21 juin 2021];9. Disponible sur: http://journal.frontiersin.org/Article/10.3389/fncir.2015.00083/abstract

45. Yamadori A. Role of the spindles in the onset of sleep. Kobe J Med Sci. 1971;17:97-111.

46. Cote KA, Epps TinAM, Campbell KB. The role of the spindle in human information processing of high-intensity stimuli during sleep. J Sleep Res. mars 2000;9(1):19-26.

47. Helfrich RF, Lendner JD, Knight RT. Aperiodic sleep networks promote memory consolidation. Trends Cogn Sci. juin 2021;S1364661321001194.

48. Beck AT, Steer RA, Ball R, Ranieri WF. Comparison of Beck Depression Inventories-IA and-II in Psychiatric Outpatients. J Pers Assess. déc 1996;67(3):588-97.

49. Beck AT, Epstein N, Brown G, Steer RA. An inventory for measuring clinical anxiety: Psychometric properties. J Consult Clin Psychol. 1988;56(6):893-7.

50. Buysse DJ, Reynolds CF, Monk TH, Berman SR, Kupfer DJ. The Pittsburgh sleep quality index: A new instrument for psychiatric practice and research. Psychiatry Res. mai 1989;28(2):193-213.

51. Horne JA, Ostberg O. A self-assessment questionnaire to determine morningness-eveningness in human circadian rhythms. Int J Chronobiol. 1976;4(2):97-110.

52. Faul F, Erdfelder E, Lang A-G, Buchner A. G* Power 3: A flexible statistical power analysis program for the social, behavioral, and biomedical sciences. Behav Res Methods. 2007;39(2):175-91.

53. Oldfield RC. The assessment and analysis of handedness: The Edinburgh inventory. Neuropsychologia. mars 1971;9(1):97-113.

54. Hoddes E, Dement W, Zarcone V. Development and use of Stanford Sleepiness Scale (SSS). Vol. 9. 1972. $150 \mathrm{p}$.

55. Ellis BW, Johns MW, Lancaster R, Raptopoulos P, Angelopoulos N, Priest RG. The St. Mary's Hospital Sleep Questionnaire: A Study of Reliability. Sleep. sept 1981;4(1):93-7. 
56. Dinges DF, Powell JW. Microcomputer analyses of performance on a portable, simple visual RT task during sustained operations. Behav Res Methods Instrum Comput. nov 1985;17(6):652-5.

57. Kleiner M. What's new in Psychtoolbox-3? :89.

58. Levitt H. Transformed Up-Down Methods in Psychoacoustics. J Acoust Soc Am. févr 1971;49(2B):467-77.

59. Leek MR. Adaptive procedures in psychophysical research. Percept Psychophys. 2001;63(8):1279-92.

60. Sterpenich V, Schmidt C, Albouy G, Matarazzo L, Vanhaudenhuyse A, Boveroux P, et al. Memory Reactivation during Rapid Eye Movement Sleep Promotes Its Generalization and Integration in Cortical Stores. Sleep. 1 juin 2014;37(6):1061-75.

61. Berry. The AASM Manual for the Scoring of Sleep and Associated Events: Rules, Terminology and Technical Specifications. Version 2.5. 2018.

62. R Core Team. R: A language and environment for statistical computing. [Internet]. na, Austria: R Foundation for Statistical Computing; 2020. Disponible sur: https://www.R-project.org/

63. RStudio Team. RStudio: Integrated Development for R [Internet]. PBC, Boston, MA: RStudio; 2020. Disponible sur: http://www.rstudio.com/

64. Benjamini Y, Hochberg Y. Controlling the False Discovery Rate: A Practical and Powerful Approach to Multiple Testing. J R Stat Soc Ser B Methodol. 1995;57(1):289-300.

65. Oostenveld R, Fries P, Maris E, Schoffelen J-M. FieldTrip: Open Source Software for Advanced Analysis of MEG, EEG, and Invasive Electrophysiological Data. Comput Intell Neurosci. 2011;2011:1-9.

66. Iber $\mathrm{C}$, Iber $\mathrm{C}$. The AASM manual for the scoring of sleep and associated events: rules, terminology and technical specifications. Vol. 1. American Academy of Sleep Medicine Westchester, IL; 2007.

67. Dube J, Lafortune M, Bedetti C, Bouchard M, Gagnon JF, Doyon J, et al. Cortical Thinning Explains Changes in Sleep Slow Waves during Adulthood. J Neurosci. 20 mai 2015;35(20):7795-807.

68. Gaudreault P-O, Gosselin N, Lafortune M, Deslauriers-Gauthier S, Martin N, Bouchard M, et al. The association between white matter and sleep spindles differs in young and older individuals. Sleep [Internet]. 1 sept 2018 [cité 12 août 2019];41(9). Disponible sur: https://academic.oup.com/sleep/article/doi/10.1093/sleep/zsy113/5025912

69. Combrisson E, Nest T, Brovelli A, Ince RAA, Soto JLP, Guillot A, et al. Tensorpac: An open-source Python toolbox for tensor-based phase-amplitude coupling measurement in electrophysiological brain signals. Schneidman-Duhovny D, éditeur. PLOS Comput Biol. 29 oct 2020;16(10):e1008302. 